\title{
Review Article \\ Regulation of Stem Cell Properties of Müller Glia by JAK/STAT and MAPK Signaling in the Mammalian Retina
}

\author{
Krista M. Beach, ${ }^{1}$ Jianbo Wang, ${ }^{1}$ and Deborah C. Otteson ${ }^{1,2}$ \\ ${ }^{1}$ Department of Basic Sciences, College of Optometry, University of Houston, Houston, TX, USA \\ ${ }^{2}$ Department of Biology and Biochemistry, University of Houston, Houston, TX, USA \\ Correspondence should be addressed to Deborah C. Otteson; dotteson@central.uh.edu
}

Received 4 November 2016; Accepted 21 December 2016; Published 17 January 2017

Academic Editor: Chuanwei Yang

Copyright (c) 2017 Krista M. Beach et al. This is an open access article distributed under the Creative Commons Attribution License, which permits unrestricted use, distribution, and reproduction in any medium, provided the original work is properly cited.

\begin{abstract}
In humans and other mammals, the neural retina does not spontaneously regenerate, and damage to the retina that kills retinal neurons results in permanent blindness. In contrast to embryonic stem cells, induced pluripotent stem cells, and embryonic/fetal retinal stem cells, Müller glia offer an intrinsic cellular source for regenerative strategies in the retina. Müller glia are radial glial cells within the retina that maintain retinal homeostasis, buffer ion flux associated with phototransduction, and form the blood/retinal barrier within the retina proper. In injured or degenerating retinas, Müller glia contribute to gliotic responses and scar formation but also show regenerative capabilities that vary across species. In the mammalian retina, regenerative responses achieved to date remain insufficient for potential clinical applications. Activation of JAK/STAT and MAPK signaling by CNTF, EGF, and FGFs can promote proliferation and modulate the glial/neurogenic switch. However, to achieve clinical relevance, additional intrinsic and extrinsic factors that restrict or promote regenerative responses of Müller glia in the mammalian retina must be identified. This review focuses on Müller glia and Müller glial-derived stem cells in the retina and phylogenetic differences among model vertebrate species and highlights some of the current progress towards understanding the cellular mechanisms regulating their regenerative response.
\end{abstract}

\section{Introduction}

In humans and other mammals, the retina, like most other regions of the central nervous system (CNS), does not spontaneously regenerate; and damage to the retina or neurodegenerative disease that kills retinal neurons results in permanent blindness. Worldwide, more than $12 \%$ of people over the age of 40 have visual impairment or blindness caused by age related macular degeneration and glaucoma, two of the neurodegenerative diseases affecting the retina $[1,2]$. As life expectancy continues to increase, the increasing prevalence of blinding neurodegenerative disease is reducing productivity and quality of life and imposing significant economic as well as social burdens to individuals, their families, and society. Current therapies can slow progression and delay vision loss but cannot restore lost vision. Consequently, there is increasing interest in identifying approaches for therapeutic retinal regeneration.

A variety of stem cells, including embryonic stem cells (ESCs), induced pluripotent stem cells (iPSCs), mesenchymal stem cells, and fetal-derived neural and retinal stem cells, are currently under investigation for regeneration and subsequent transplantation of retinal neurons (see reviews in [310]). With advancements in gene editing using CRISPR/Cas9 technologies and the ability to expand cells in culture prior to differentiation, extrinsic sources such as ESCs and iPSCs are promising for developing strategies to correct preexisting genetic defects in vitro [11]. However, there are potential ethical concerns with the use of ESCs or progenitors from embryonic or fetal tissues, making them less attractive for therapeutic regeneration. Further, extrinsic stem cells will require surgical transplantation and integration of new neurons into existing circuitry. Although the retina is normally an immune privileged tissue, retinal damage and degenerative disease compromise the blood/retinal barrier, allowing ingress of immune cells [12-15]. Therefore, transplantation therapies may also require immunosuppression for long-term viability of the engrafted cells. An intrinsic retinal stem cell would alleviate concerns of integration and immune response and 


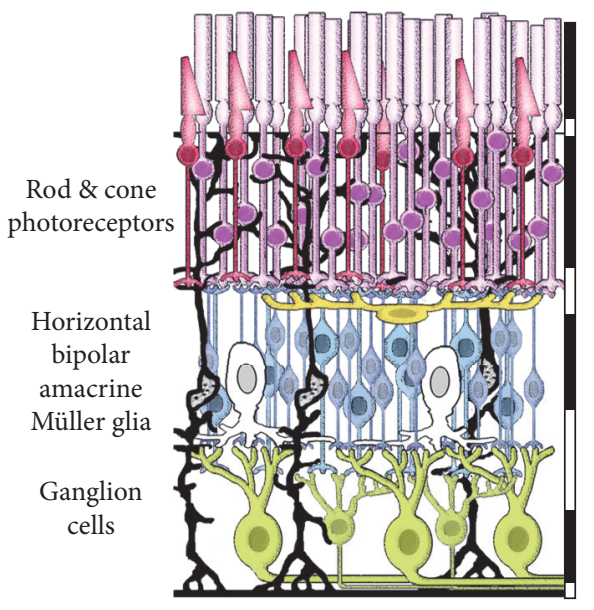

(a)

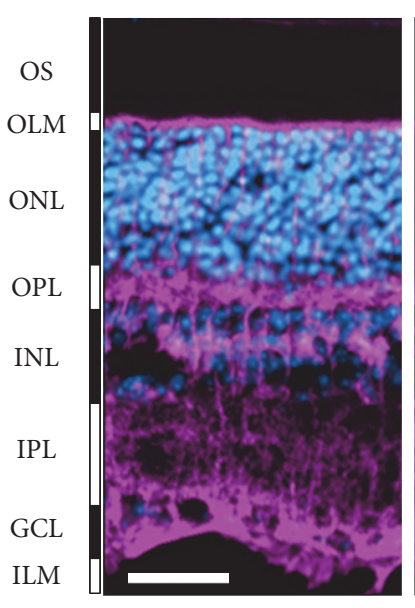

(b)

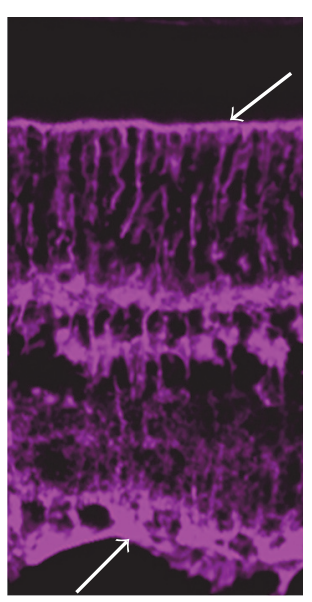

(c)

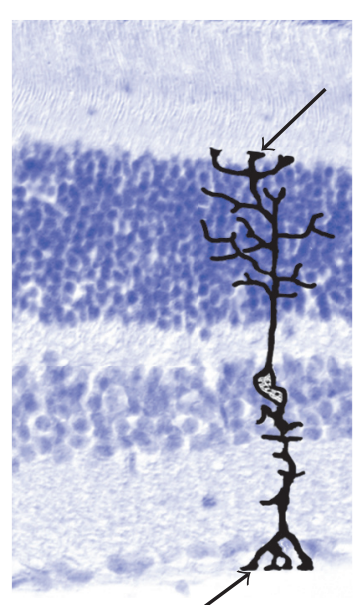

(d)

FIGURE 1: Retinal structure and cellular organization. (a) Diagram shows organization of retinal neurons and Müller glia. The cell bodies of rod (purple) and cone (red) photoreceptors are in the outer nuclear layer (ONL) and the photoreceptor outer segments (OS) contain the photopigments that absorb light. Rod and cone bipolar (blue), horizontal (yellow), and amacrine (white) cells are in the inner nuclear layer (INL), with retinal ganglion cells (green) located in the ganglion cell layer (GCL). Between the nuclear layers are the outer and inner plexiform layers (OPL, IPL) containing the synaptic terminals. Müller glia (black) have cell bodies located in the INL and extend processes throughout the retina. (ILM, OLM). (b, c, d) Photomicrographs of adult mouse retina. (b, c) Müller glia are immunostained for glutamine synthetase (magenta) revealing their radial processes that extend the full thickness of the retina. The Müller glial endfeet form the inner limiting membrane (ILM) and outer limiting membrane (OLM) (arrows). (d) Photomicrograph showing histology of adult mouse retina stained with toluidine blue, showing retinal lamina and overlaid with a diagram of a Müller glial cell. Arrows indicate glial endfeet at ILM and OLM. Scale bar = 50 microns in (b), (c), (d).

would provide an alternative strategy to complement the use of extrinsic stem cells.

Müller glia are intriguing candidates for intrinsic retinal stem cells. Müller glia are radial glial cells within the retina and are generated from the same lineage as retinal neurons. In the mature retina, Müller glia maintain retinal homeostasis, buffer ion flux associated with phototransduction, and form the blood/retinal barrier within the retina proper. Although they contribute to gliotic responses and scar formation following retinal injury, Müller glia also show regenerative capabilities that vary across species. This review focuses on Müller glia and Müller glial-derived stem cells in the retina and the phylogenetic differences among model vertebrate species and highlights current progress towards understanding and harnessing their regenerative response.

\section{Retinal Structure and the Origin of Müller Glia}

The retina is a thin layer of neural tissue located at the posterior pole of the eye. It consists of (a) photoreceptors (rods and cones) that convert light stimuli into neurochemical signals, (b) three major classes of interneurons (horizontal, amacrine, and bipolar cells) that perform initial information processing, (c) Müller glia that perform a multitude of support functions, and (d) projection neurons (retinal ganglion cells) that extend axons through the optic nerve and optic tract to convey the visual image information to higher processing centers within the brain [16-18]. The retinal cells are organized in a highly ordered laminar structure (Figure 1), which allows identification of cell types by position, morphology, and gene expression. The retina is developmentally part of the CNS. Lineage analysis has shown that the multipotent retinal progenitors that make up the embryonic retinal neuroepithelium generate all types of retinal neurons, as well as the Müller glia [16, 19, 20]. Apart from its importance in vision, the neural retina serves as a model system for studying the CNS, as it is the only portion of the central nervous system located outside of the cranium and can be noninvasively imaged and functionally tested in vivo. The process of retinal regeneration recapitulates many aspects of retinal development, with similar patterns of gene expression, cell fate specification, and the order of neurogenesis.

\section{Müller Glia: Stem Cells for Retinal Regeneration in Fish}

The initial evidence for the stem cell characteristics of Müller glia came from research to discover the cellular source of ongoing neurogenesis and regenerative responses in the retinas of fish. The capacity for neurogenesis in the mature retinas of fish appears to be teleologically related to their overall pattern of indeterminate growth and the associated continuous growth of their eyes. Eye growth in fish results in large part 
from a general expansion/stretching of the retina, leading to decreasing retinal density for most types of retinal neurons within the central retina [21]. There is also ongoing neurogenesis, which occurs at two sites: (1) the circumferential germinal zone (CGZ), where a population of retinal progenitors persists and continually adds concentric rings of new neurons to the retinal margin, and (2) the central retina, where new rods are added continually to the existing photoreceptor mosaic [22]. Neurogenesis of rod photoreceptors in the fish retina begins after hatching and continues throughout life [23-28], with new rod photoreceptors generated from a population of slowly proliferating, PAX6-expressing progenitors within the inner nuclear layer (INL) [29]. These INL progenitors arise from the Müller glia and continue to divide as they migrate to the outer retina to become rod precursors, which subsequently differentiate into rod photoreceptors $[26,28$, 29]. Ongoing neurogenesis in the fish retina appears to keep the Müller glia poised to respond to retinal damage by initiating intrinsic neurogenic programs.

Retinal regeneration in fish has been studied for over 50 years [30-34]. A variety of traumatic, surgical, neurotoxic, or phototoxic injuries have been used to induce regenerative neurogenesis, and the outcome is a fully laminated retina, albeit with some relatively minor organizational differences, and a restoration of circuitry and function [34-39]. Although multiple studies had reported clusters of mitotically active cells in the INL following retinal injury [40, 41], the source of the new retinal cells was not identified as Müller glia until 2007 [40-42]. Regardless of the injury paradigm, there appear to be several stages to the retinal response [43]. There is an initial, nonproliferative stage that occurs during the first 2 days after injury, during which Müller glia transiently upregulate expression of the intermediate filament, glial fibrillary acidic protein (GFAP), a key marker of gliosis $[44,45]$. The gliotic response of Müller glia is not prominent, and the subsequent, regenerative response in zebrafish begins with an initial, limited proliferation of the Müller glia and generation of a pool of Müller glial-derived progenitors in the INL [4649]. The Müller glial-derived progenitors continue to proliferate, forming columns of dividing cells that span the retinal layers and, around 7 days after injury, differentiate into retinal neurons [46, 50-52]. Interestingly, the switch from gliosis to proliferation is critical for the regenerative response in zebrafish, as blocking injury-induced proliferation of Müller glia, using 5-fluorouracil or morpholinos against PCNA, enhances the gliotic response, resulting in robust and persistent upregulation of GFAP, and prevents injury-induced neurogenesis [45]. Müller glial-derived progenitors are capable of regenerating all neuronal cell types in an injured retina and restoring visually guided behaviors [34-36, 38, 39, 53-58]. Although early studies suggested that only those subpopulations of neurons that were lost as a result of the initial injury were regenerated $[49,59,60]$, recent evidence shows that, even in cases of localized injury to one cell type [e.g., phototoxic injury to photoreceptors or N-methyl-D-aspartate (NMDA) injury to retinal ganglion cells], additional cell types can be produced [48].

\section{Müller Glia: Gliosis and Injury Response in Mammals and Birds}

In warm blooded species, spontaneous regeneration by Müller glia does not occur to any appreciable extent in vivo, leading to questions about the designation of Müller glia as retinal stem cells in these species, particularly in mammals [64]. In the mammalian retina, the primary glial response to retinal injury is gliosis, which is characterized by robust upregulation of GFAP, limited (if any) proliferation, cellular hypertrophy, and formation of glial scars (reviewed in [65, 66]; see also [67-72]). In posthatch birds, there is a slightly more robust response to retinal injury, with gliosis accompanied by a limited neurogenic response that declines with age $[64,73-75]$.

Evidence for neurogenesis by Müller glia in vivo following acute retinal injury has been demonstrated in posthatch chick, rat, and mouse $[62,64,71,73-77]$ but requires manipulation of various exogenous factors (see below) or overexpression of neurogenic or proneural genes such as Asclla $[78,79]$ and Atoh7 [80, 81]. Intraocular injection of selective neurotoxins that kill either photoreceptors or ganglion/amacrine cells in the adult rodent eye generates large numbers of reactive Müller glia but little proliferation $[62,82]$. The proliferative response is enhanced by intraocular injection of a variety of extrinsic growth factors, including CNTF, EGF, FGF1, FGF2, and insulin in posthatch chickens [73-75] and in rodents $[62,71,83,84]$. However, the overall extent of the neurogenic capacity of mammalian or avian Müller glia is low, even with growth factor stimulation, and, in the mammalian retina, low numbers of neuronal cells are generated and the majority of proliferating progenitors fail to survive in the long term [62]. A better understanding of the mechanisms that promote the proliferative and neurogenic responses is needed before clinically relevant levels of regeneration are achieved in vivo.

\section{Mechanisms Regulating Gliosis versus Neurogenesis: JAK/STAT versus MAPK Signaling}

Promoting clinically relevant levels of regeneration from Müller glia in the mammalian or human retina will require suppression of gliosis and enhancement of their proliferative and neurogenic responses. Multiple signaling molecules and their downstream signal transduction cascades have been implicated in regulating the injury and regenerative responses in the retina, including notch [85-88], tumor necrosis factor alpha (TNF- $\alpha$ ) [89], transforming growth factor beta (TGFß) $[84,90-92]$, insulin $[70,73]$, midkine (MDKN) [93-95], ciliary neurotrophic factor (CNTF) [75, 92, 96-99], epidermal derived growth factor (EGF) $[62,74,84,100]$, and fibroblast growth factors (FGFs) [62, 75, 101, 102]. Many of these signaling pathways converge on JAK/STAT (Janus kinase/signal transducer and activator of transcription) and MAPK (mitogen-activated protein kinase) signal transduction cascades (Figure 2). Retinal injury stimulates release of a variety of cytokines and mitogens, including EGF, FGF1, FGF2, and 


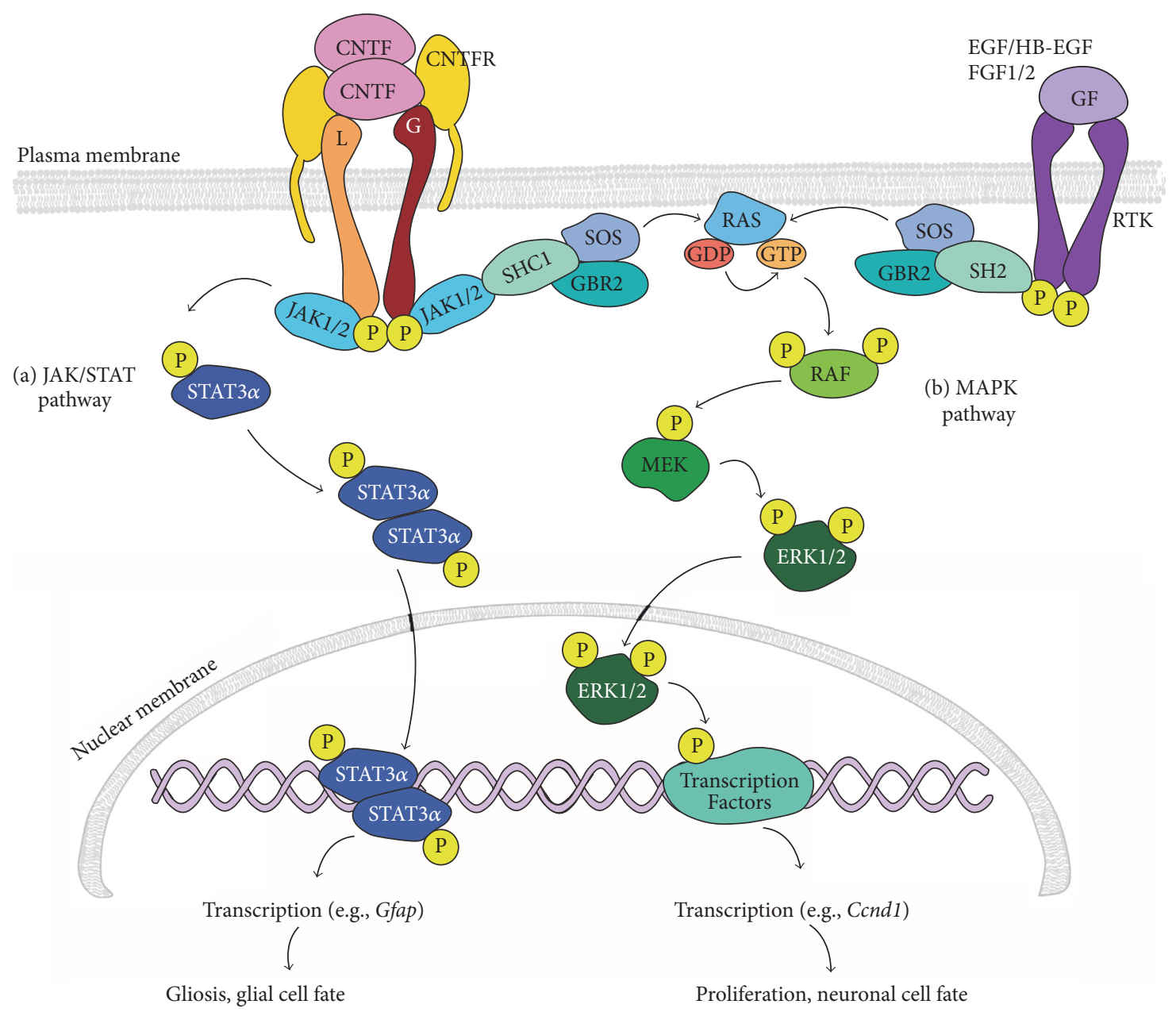

FIGURE 2: Summary diagram of JAK/STAT and MAPK signal transduction. (a) To activate JAK/STAT signaling, CNTF binding to the GPI linked CNTFR $\alpha$ initiates recruitment of LIFR $\beta(\mathrm{L})$ and GP130 (G) to form the hexameric CNTF receptor complex. Recruited LIFR $\beta$ and GP130 are phosphorylated on their cytoplasmic domain by JAK1/2. Activated JAK1/2 phosphorylate STAT3 $\alpha$, which forms homodimers that translocate to the nucleus. pSTAT3 homodimers bind to DNA and activate transcription of target genes, such as Gfap, to initiate gliosis. (b) The MAPK signaling pathway can be activated downstream of ligand binding to receptor tyrosine-kinases (RTKs) by growth factors including HB-EGF, EGF, FGF1, and FGF2 or by activation of CNTFR by CNTF. In both pathways, adaptor proteins such as GBR2 recruit SOS to the activated receptor, and subsequent activation of SOS leads to phosphorylation of RAS, MEK, and finally ERK1/2. Activated ERK1/2 translocate into the nucleus and phosphorylate several transcription factors involved in cell proliferation, cell survival, and cell differentiation.

CNTF, which have been implicated in various aspects of glial activation and proliferation [103-106]. However, endogenous expression levels of growth factors and cytokines following injury are insufficient to promote significant proliferation of mammalian Müller glia. Injury-induced proliferation can be enhanced by intraocular injection of EGF, FGF1, FGF2, and CNTF, either alone or in various combinations with each other or with insulin [62, 70, 73-75, 92, 98, 101, 107]. All of these factors can activate intracellular signal transduction cascades via JAK/STAT and/or MAPK signaling pathways. Therefore, examination of these signaling pathways and how their activation relates to gliosis and retinal regeneration in fish, birds, and mammals is important to begin to understand the mechanisms contributing to the differential injury responses.
Intracellular signaling through the JAK/STAT pathway is activated by receptor binding of a variety of ligands, including cytokines (e.g., interleukins [108], interferons [109], and CNTF [110]), growth factors (e.g., EGF [111], FGF [112]), and hormones (growth hormone [113], thyrotrophin stimulating hormone [114]). In JAK/STAT signaling, ligand binding to cognate receptors results in phosphorylation of receptorassociated JAK1/2, which causes rapid (within minutes) phosphorylation of STAT3 and a delayed (within hours) phosphorylation of STAT1 $[115,116]$. Phosphorylated STAT3 (pSTAT3) and pSTAT1 form homodimers or heterodimers via phosphotyrosyl peptide interaction of their SH2 (Src homology 2) domains, resulting in translocation to the nucleus, binding to DNA at the consensus binding sequence TTCC $[\mathrm{C} / \mathrm{G}] \mathrm{GGAA}$, and transcription of target genes [116]. 
CNTF can activate MAPK signaling downstream of its receptor; however activation of MAPK signaling more typically occurs downstream of receptor tyrosine-kinase activation by a variety of ligands, including EGF, FGFs, and insulin. In the MAPK signaling cascade, binding of a signaling ligand to its receptor causes a series of sequential phosphorylation reactions. Each step in the cascade can be performed by multiple proteins, making the cascade both diverse and complex (see reviews in $[117,118]$ ). Briefly, phosphorylation of the cytoplasmic domains of the cytokine receptors causes adaptor molecules to recruit proteins that activate RAS (rat sarcoma oncogene), which phosphorylates RAF (rapidly accelerated fibrosarcoma), which phosphorylates MAPK kinases (including MAPK/ERK kinases 1 and 2; a.k.a. MEK1/MEK2), which phosphorylate MAPKs, including extracellular signalregulated protein kinase (ERK), c-Jun-N-terminal kinase (JNK), and protein 38 (p38). Phosphorylated MAPK translocates to the nucleus and phosphorylates a variety of transcription factors that activate target gene transcription.

\section{CNTF Activation of JAK/STAT and Gliosis}

Among the factors that can activate JAK/STAT and MAPK signaling, CNTF plays multiple roles in the retinal injury response and particularly in activating the gliotic responses of Müller glia. CNTF expression is upregulated in Müller glia following a variety of retinal injuries in the zebrafish [98], posthatch chicken [75], and mammals [103, 104, 106, 119]. Müller glia of all species express little, if any, GFAP in the absence of injury, neurodegenerative disease, or other insults $[67,120,121]$. However, intraocular injection of CNTF into otherwise uninjured eyes of zebrafish [75], posthatch chickens [92], mice [99], and rats [115] increases GFAP in Müller glia, mimicking a gliotic response [98]. Upregulation of GFAP by CNTF is mediated by STAT3 signaling via direct binding of phosphorylated STAT3 (pSTAT3) dimers to the GFAP promoter $[99,122,123]$. Consistent with a role in gliosis, STAT3 expression also increases in Müller glia following ouabain- or light-induced injury in zebrafish $[47,98]$, and STAT3 phosphorylation is similarly increased in Müller glia following injury to the avian [75] and mouse retina $[124,125]$.

\section{JAK/STAT and MAPK Regulation of Müller Glial Proliferation}

In addition to activating JAK/STAT signaling, injury activates MAPK signaling in Müller glia in zebrafish and mice [98, 126]. Various growth factors, including EGF, FGFs, and insulin, activate MAPK signal transduction directly downstream of tyrosine-kinase receptors, whereas CNTF activates MAPK via JAK activation of SHP2/RAS (Figure 2) [98, 127, 128]. Following a penetrating injury, combinatorial treatments of insulin combined with either heparin binding EGFlike growth factor (HB-EGF; an EGF-related but more potent mitogen [129]) or FGF2 increase proliferation of Müller glia in the zebrafish retina, an effect that is reduced by inhibition of MAPK or JAK/STAT [74, 100, 101]. Similarly, in both avian and mouse retinas, NMDA injury increases pERK and pSTAT3 in Müller glia $[74,75,115]$. Proliferation of Müller glia is increased by exogenous HB-EGF, FGF2, insulin, or combinations of CNTF and FGF2, in NMDA injured, but not in uninjured, retinas [75]. Although phosphorylation of ERK1/2 and STAT is increased by these same factors, the ability of CNTF/FGF2 to increase proliferation of Müller glial-derived progenitors in NMDA injured chicken retinas requires activation of JAK/STAT $[74,130]$. Similarly, the proliferative effects of CNTF can be blocked at the receptor level by inhibition of the gp-130 coreceptor or postreceptorally by inhibition of either JAK2 phosphorylation or STAT3 dimerization/nuclear translocation [75, 99]. If JAK/STAT signaling mediates both gliosis and proliferation of Müller glia in response of CNTF or injury, a key question is how to promote proliferation of Müller glia-derived stem cells to repopulate areas of retinal degeneration, without also inducing gliosis.

\section{Müller Glia In Vitro}

Despite similarities between fish, birds, and mammals in the Müller glial response to exogenous growth factors, modulation of JAK/STAT and MAPK signaling in the mammalian retina remains insufficient to stimulate clinically relevant regenerative responses. There is evidence that both intrinsic and extrinsic mechanisms contribute to inhibition of the neurogenic/regenerative response of Müller glia in the retinas of higher vertebrates. Although regeneration in the fish retina can occur throughout life, there is an age-associated decline in the proliferative response of Müller glia to retinal injury and exogenous mitogens in rodents $[83,131]$. The genetic background in different mouse strains also has significant influence on the ability of Müller glia to proliferate in response to injury and exogenous growth factors, although specific genetic factors have yet to be identified [132]. Since intraocular injection of exogenous factors can promote Müller glial proliferation, it is likely that there are insufficient endogenous levels of known growth factors. In addition, there are likely additional, unidentified factors that are required to activate key signaling pathways or that actively inhibit the proliferative and regenerative capacity in vivo. Consistent with this, isolated mammalian Müller glia show increased proliferation and neurogenic potential in vitro. This may reflect the greater ability to manipulate exogenous factors in vitro, as well as the removal of the Müller glia from any local inhibitory signals. Thus, even though the ultimate goal for using Müller glial-derived progenitors is to stimulate neural regeneration in situ, the use of primary Müller glia and permanent cell lines offers an opportunity to examine mechanisms underlying their proliferative and regenerative responses under more controlled conditions in vitro.

Several Müller glial cell lines have been described: ImM10, conditionally immortalized Müller glia from P10 mouse retinas [61, 133]; MIO-M1, spontaneously immortalized Müller glia from adult human retinas [134]; rMC1, SV-40 immortalized Müller glia from light-injured rat retinas [135]; TR-MUL5, conditionally immortalized Müller glia from rat [136]; and MU-PH1, conditionally immortalized Müller glia from 2-month-old mice. Direct comparisons are complicated by differences in the age and species of origin, variable culture conditions, and the panels of genes that 
have been analyzed in different studies. However, in standard culture conditions, all express at least some genes typical of Müller glia in vivo (e.g., vimentin, Sox2), as well as nestin, an intermediate filament typically associated with neural stem cells. Only rMC-1 has robust GFAP expression, consistent with its origin from light-injured retinas [135], although one study also reported low levels of GFAP immunoreactivity in MIO-M1 cells [137]. There are concerns that overexpression of oncogenes will fundamentally change the identity and cellular responses of Müller glial cell lines. The conditionally immortalized cell lines, ImM10 (mouse) and TR-MUL5 (rat), contain an inducible, temperature sensitive SV40T-antigen, thereby allowing elimination of oncogene expression under appropriate conditions.

There is a temporal change in cell cycle kinetics of primary Müller glia, which initially proliferate slowly, even in the presence of serum containing medium [133]. After continued culture, primary Müller glia become more highly proliferative, consistent with spontaneous immortalization $[133,134]$. Within the first two weeks in culture, Müller glia change morphology and downregulate key genes associated with glial function [e.g., glutamine synthetase (GS), cellular retinaldehyde-binding protein (CRALBP/RLBP1)] [138]. We observed reduced proliferation of ImM10 cells when grown in serum-free medium with nonimmortalizing conditions, although rates of proliferation increased and the differences between immortalizing and nonimmortalizing conditions diminished at higher passage numbers [133]. Thus, careful comparative studies of cultured Müller glia with their in vivo counterparts will be needed for final validation of any identified mechanisms regulating proliferation and neurogenic competence.

Immortalized Müller glia can be induced to generate cells expressing neuronal genes [61, 134, 139-142]. ImM10, MIO-M1, and MU-PH1 generate neurospheres in response to specific growth factors, typically a combination of epidermal derived growth factor (EGF), fibroblast growth factor 2 (FGF2), and/or insulin (Figure 3(b)), and upregulate a variety of genes typical of retinal progenitors, including Pax6 and nestin $[61,134,137,139]$. Using a variety of in vitro differentiation protocols, Müller glial-derived progenitors from neurospheres will alter their morphology to resemble cultured neurons, showing condensed nuclei and long, branching neuritelike processes (Figure 3(d)). Redifferentiated human Müller glial-derived progenitors express markers of most retinal cell types and have been shown to respond to light [137]. Transplantation of in vitro differentiated photoreceptors from Müller glial-derived progenitors partially restored light response in a rat model of rapid photoreceptor degeneration, as measured by increases in the a-wave of the electroretinogram (a measure of photoreceptor function) [140]. Transplantation of in vitro differentiated Müller glia into rat retinas, following pharmacological depletion of retinal ganglion cells, partially restored the negative scotopic threshold response of the electroretinogram (an indicator of retinal ganglion cell function) [139]. However, despite the presence of the newly generated cells in their appropriate lamina and evidence of some synapse formation with upstream neurons, the new cells failed to extend axons into the optic nerve or connect to visual centers in the brain. In all differentiation paradigms reported, relatively large numbers of cells continue to express glial genes and retain a glial morphology. Additionally, the number of neurons generated is relatively low, their morphology is inconsistent, and gene expression profiles have yet to demonstrate expression of all genes necessary for specification and functional maturity of individual retinal cell types.

\section{JAK/STAT and MAPK in Müller Glia In Vitro}

There have been few systematic studies of the activity of specific signal transduction cascades in Müller glia in vitro. Since activation of JAK/STAT or MAPK signaling in Müller glia can regulate gliosis and proliferation, we analyzed activation of STAT3 and MAPK pathways in ImM10 cells by western blot. There were no changes in total STAT3 or PSTAT3 in neurosphere or differentiation cultures; and, unexpectedly, despite the presence of both EGF and FGF2 in sphere forming media, there was no change in pERK1/2 in neurospheres [143]. In contrast, $\mathrm{pERK} 1 / 2$ increased in ImM10 cells in differentiation conditions despite the absence of exogenous EGF or FGF2 [143]. However, EGF and FGF2 mRNA levels are upregulated in differentiation cultures of ImM10 cells [61], suggesting that endogenously produced factors contribute to activation of MAPK signaling in these cells.

During neonatal retinal development, CNTF has been proposed to modulate a "molecular switch" that promotes either a glial or neuronal cell fate via concentration dependent activation of STAT3 versus MAPK signaling, respectively $[144,145]$. In rat P1 retinal explants and dissociation cultures, low concentrations of CNTF $(<50 \mathrm{ng} / \mathrm{ml})$ increase the number of cells expressing neuronal genes via MAPK signaling, whereas high concentrations $(100 \mathrm{ng} / \mathrm{ml})$ increase the number of cells expressing glial genes via STAT3 signaling [144]. Inhibition of STAT3 signaling abolishes CNTF's repression of photoreceptor markers in mouse retinal progenitor cell cultures $[146,147]$, whereas inhibition of MAPK signaling abolishes the increase in the number of cells expressing neuronal markers in dissociation cultures of neonatal rat retina treated with low concentrations of CNTF [144].

To test if low levels of CNTF could promote neurogenesis in differentiation cultures of ImM10 cells, we analyzed gene expression following addition of low concentrations of CNTF $(20 \mathrm{ng} / \mathrm{ml})$ [61]. Consistent with our previous study, genes associated with multiple neuronal types were detected in differentiation cultures, including photoreceptors (Rhodopsin, Opn1sw, and Nr2e3), retinal ganglion cells (Sncg, L1Cam), and bipolar cells (Prkca). However, addition of CNTF did not alter the overall patterns or levels of gene expression [143]. In addition, GFAP was not detected in differentiation cultures, either with or without CNTF, suggesting that there was no enhancement of gliosis. Surprisingly, CNTF did not change phosphorylation of STAT3 or ERK in ImM10 cells, reflecting the failure of CNTF to activate either JAK/STAT or MAPK signaling. These findings suggest that the ImM10 cells in vitro respond to CNTF differently than Müller glia in vivo. One potential explanation is that the effects of CNTF may require additional 


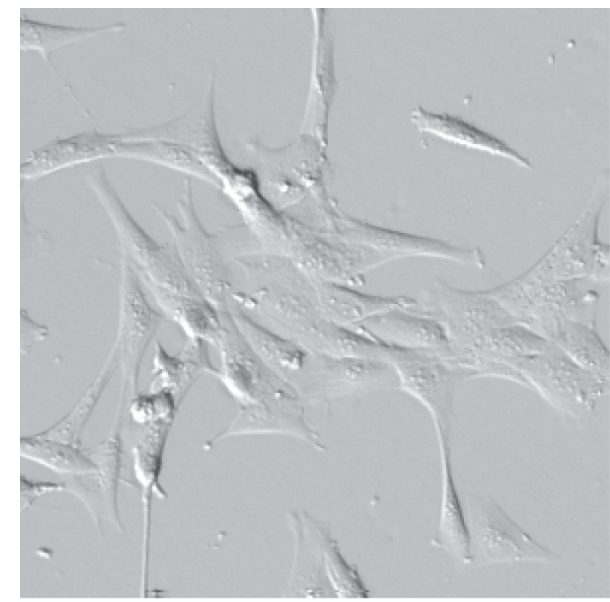

(a)

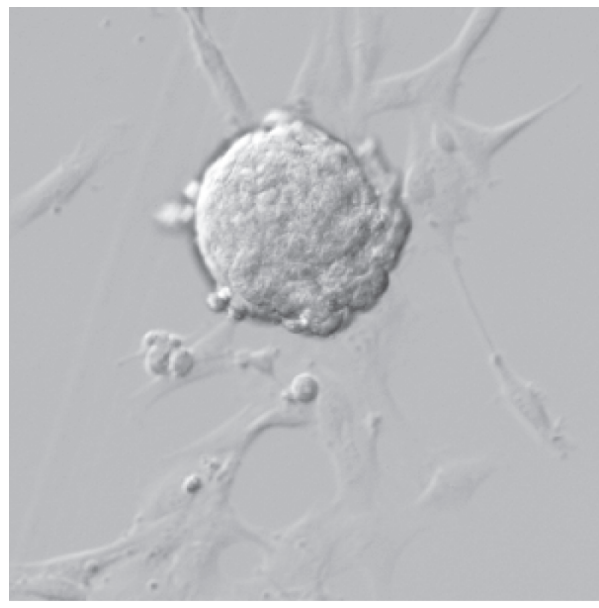

(c)

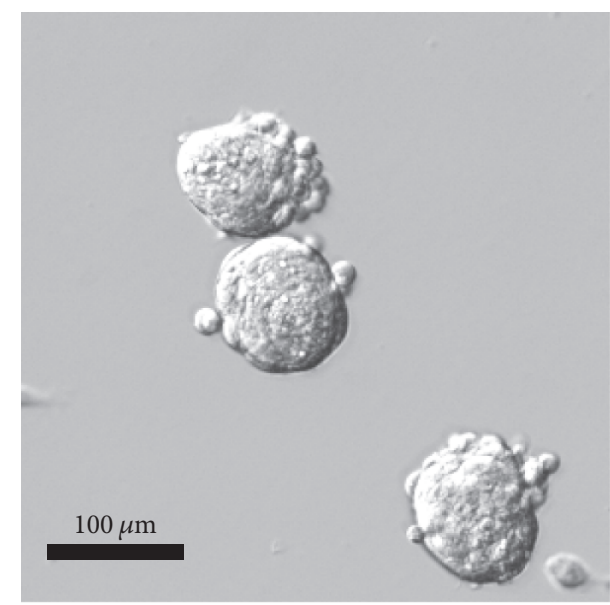

(b)

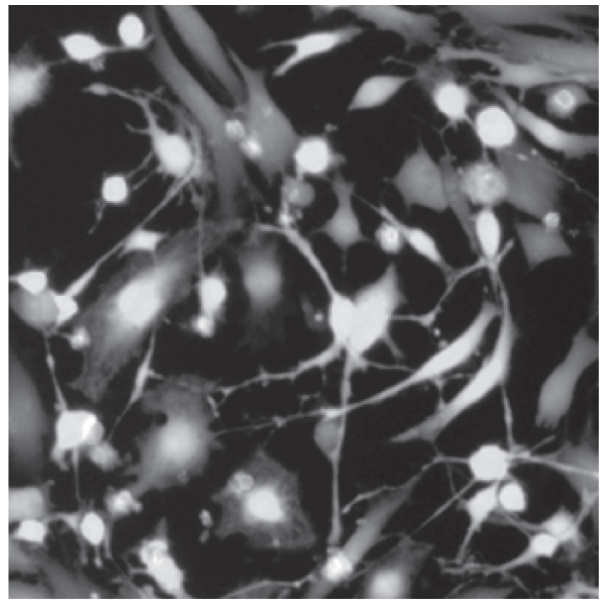

(d)

FIGURE 3: Morphology of mouse Müller glia in growth, neurosphere, and differentiation cultures in vitro. Conditionally immortalized mouse Müller glia (ImM10 cell line) at different stages of in vitro differentiation as previously described [61]. (a) ImM10 cells in growth media (Neurobasal, 2\% fetal bovine serum, B27 supplement, and penicillin/streptomycin) under immortalizing conditions $\left(33^{\circ} \mathrm{C}, 50 \mathrm{U} / \mathrm{ml}\right.$ interferon gamma) show typical morphology of cultured Müller glia. (b) ImM10 cells following 4 days in sphere forming medium (Neurobasal, B27

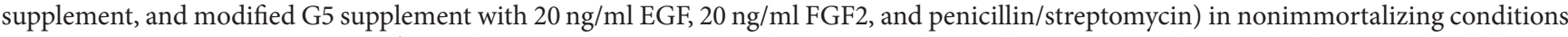
to prevent T-antigen expression $\left(39^{\circ} \mathrm{C}\right.$, without interferon gamma), showing typical nonadherent neurospheres. (c) Spheres at 1 day following transfer to priming medium (Neurobasal, G5 supplement modified to contain EGF ( $20 \mathrm{ng} / \mathrm{ml}$ ) but without FGF2, and penicillin/streptomycin; nonimmortalizing conditions), neurospheres adhere to plate, and cells begin to migrate onto dish. (d) Following priming, ImM10 cells in differentiation medium (Neurobasal, B27, and pen/strep; nonimmortalizing conditions) for 2 days and stained with CalceinAM show variable morphologies and include cells with distinct neuronal morphology (small cell body, multiple thin processes).

cells or factors not present in the cell line. Consistent with this idea, stem cells from dental pulp treated with conditioned media from injured rat retinas upregulated rhodopsin expression in vitro, whereas those treated with conditioned media from purified Müller glia did not [148].

The JAK/STAT and MAPK signaling pathways are implicated in retinal cell fate choice, proliferation, and gliotic hypertrophy after retinal injury and can be activated by a variety of growth factors including EGF, HB-EGF, FGF2, insulin, and CNTF. The JAK/STAT pathway appears to mediate both beneficial (proliferation) and detrimental (hypertrophy/ gliotic) aspects of the injury response in vivo (Figure 4). Our findings that MAPK activation increased in ImM10 cells cultured under conditions that increased neuronal gene expression are consistent with a role for MAPK signaling in mediating a neuronal cell fate over glial fate choice. In mammalian retinal injury, the gliotic response predominates over the regenerative response, although the regenerative response can be enhanced with the addition of exogenous growth factors and cytokines that activate JAK/STAT and MAPK signaling. Unfortunately, this enhancement is still insufficient to make regeneration predominate over gliosis.

\section{Other Mechanisms}

Although activation of JAK/STAT and MAPK signaling by CNTF, EGF, and FGFs can promote proliferation and the 


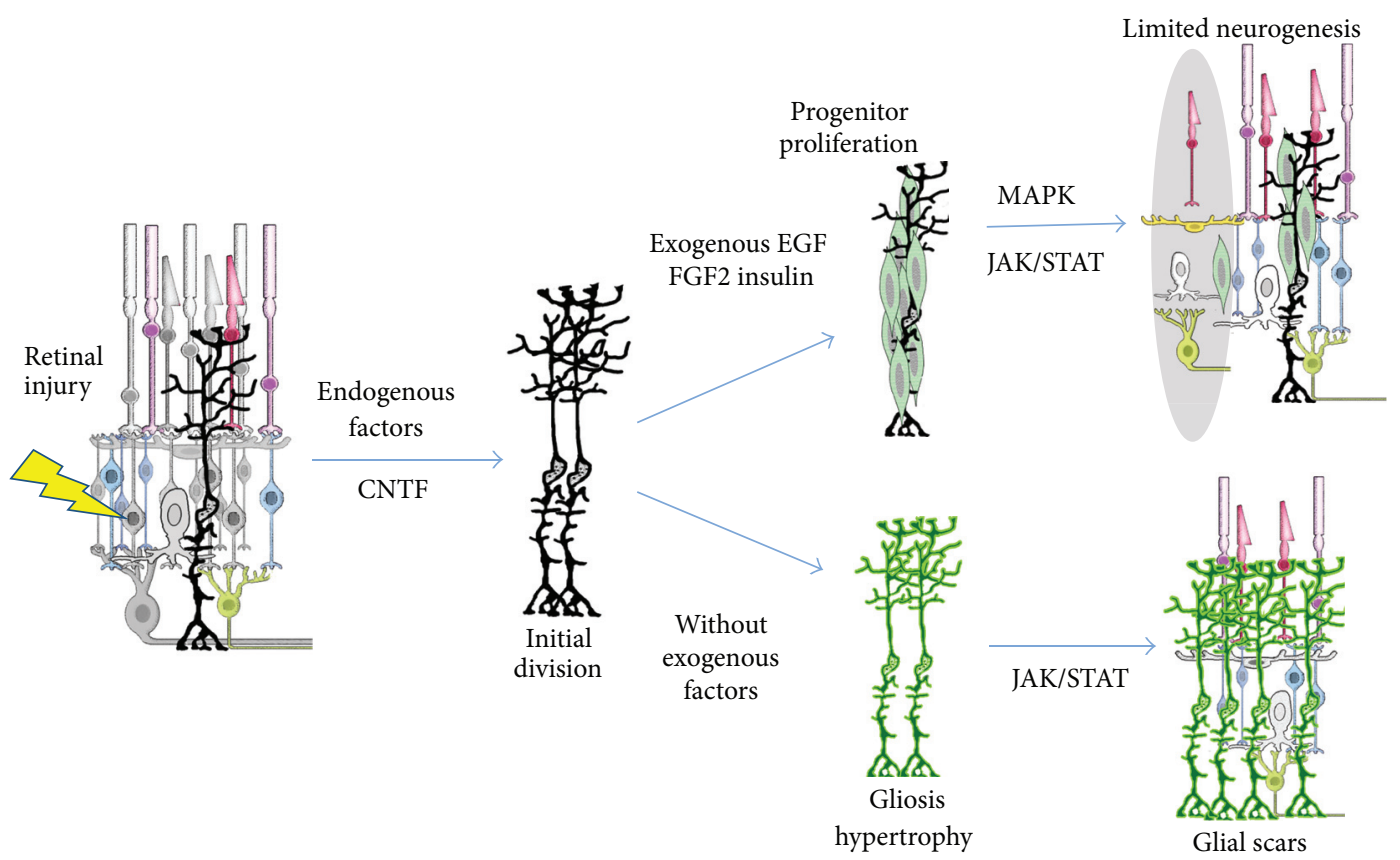

Figure 4: The proposed involvement of CNTF, JAK/STAT, and MAPK signaling in gliosis and neural regeneration by Müller glia in mammalian retina. Retinal injury (lightning bolt) kills retinal cells (gray cells) and stimulates release of growth factors, including CNTF, resulting in limited cell division of Müller glia (MG). In the absence of exogenous growth factors, increased JAK/STAT signaling (lower arrows) in activated MG promotes gliosis (bright green), resulting in glial scars, but neurons are not regenerated. Activation of MAPK and JAK/STAT signaling by exogenous factors, including EGF, FGF2, and insulin (upper arrows), produces proliferative progenitors (light green), which can regenerate some retinal neurons (in gray shaded oval), such as amacrine cells [62] and photoreceptors [63]. Some undifferentiated progenitor cells (light green) persist following resolution of the regenerative response. Even with exogenous factor stimulation, mammalian retinas fail to restore all lost cells.

glial/neurogenic switch, these signal transduction pathways do not act in isolation. Rather, they function within the context of a wide variety of other cellular mechanisms that contribute to the retinal injury response and regeneration in various contexts. Extensive discussion of all the cellular mechanisms regulating the injury and regenerative responses of Müller glia is beyond the scope of this article; several recent reviews can provide more information [22, 64, 149-154]. In addition to its roles in regulating gliosis and cell fate specification, CNTF is also implicated in neuroprotection and axonal outgrowth through activation of the MAPK, JAK/STAT, and/or PI3K (phosphatidylinositol-3 kinase) pathway [155159]. Other pathways that are important in regulating cell cycle reentry and exit, gliosis, neurogenesis, and differentiation of Müller glia following retinal injury include the following: notch $[85,87,160]$ and WNT signaling $[161,162]$; activation of a variety of signal transduction cascades by TNF- $\alpha[87$, 89], TGF- $\beta$ [163], and BMP/SMADs [84, 91, 164]; regulation of cell cycle by $c c n d 1$ and $p 27$ (kip1) $[83,85,165]$; microRNAs [78, 166-168]; and the effects of transcriptional regulators that regulate retinal development, such as the proneural gene Asclla $[79,142,162]$ and the neurogenic gene Atoh7 [80, 81, 141]. Thus, expanding our understanding of how multiple pathways integrate to regulate the injury and regenerative responses of Müller glial will be important for continued progress in the field.
Analysis of retinal regeneration in fish has identified a variety of signaling molecules and their downstream signal transduction cascades that have shown promise for enhancing regenerative responses of Müller glia and warrant continued study. One intriguing, yet understudied, molecule that plays a role in retinal neurogenesis and regeneration in zebrafish is midkine (MDKN) [169]. MDKN is a heparin binding protein that interacts, either directly or indirectly, with a number of receptors, including ALK, LRP, notch, and protein tyrosine phosphatase-zeta (PTP- $\xi$ ), to modulate downstream signal transduction cascades [94, 169-174]. Binding of MDKN, or the structurally related pleiotrophin, to PTP$\xi$ blocks its phosphatase activity, resulting in increased phosphorylation of a variety of tyrosine-kinase receptors and their substrates and potentiating downstream signal transduction cascades [94]. In zebrafish, $\mathrm{MDKNa} / \mathrm{MDKNb}$ are expressed in both retinal progenitors and Müller glia and are upregulated following retinal injury $[93,95]$. Morpholino inhibition of MDKNa reduces proliferation of Müller glial-derived progenitors and limits regeneration of rod photoreceptors following light damage in zebrafish [93]. Much less is known about MDKN in the mammalian retina, although it is neuroprotective for rod photoreceptors following light damage in mice [175] and rats [176]. We found that Mdkn mRNA is upregulated in ImM10 cells under differentiation condition in vitro [61]. Given the pleiotropic effects of MDKN and its 
potential to modulate multiple signal transduction cascades that impact the proliferative and neurogenic responses of Müller glia, it would be interesting to assess combinatorial effects of MDKN and growth factor stimulation of JAK/STAT, MAPK, or other signal transduction cascades on Müller glial proliferation and retinal regeneration.

\section{Conclusions}

Müller glia are particularly appealing as a cellular source for retinal regeneration because they are intrinsic to the retina and offer the potential to regenerate neurons in situ, without transplantation. Despite a growing body of research showing the neurogenic potential of Müller glia in the mammalian retina, a level of regenerative response sufficient for potential clinical applications has yet to be achieved. Given the overall modest outcomes to date, cultured Müller glia seem unlikely to provide a clinically relevant path for generating sufficiently large numbers of retinal progenitors for transplantation. Successful regenerative strategies using transplantation are more likely to build on the ongoing progress in generating retinal progenitors and neurons from other sources, such as induced pluripotent stem cells [3-6, 15, 177-184]. Therefore, the importance of studies to promote in vitro differentiation of Müller glia lies in the ability to manipulate the cellular environment and to dissect cellular mechanisms that regulate their regenerative responses. Nevertheless, there is clear evidence that Müller glia change patterns of proliferation and gene expression as they adapt to culture conditions and that the ability of Müller glia to respond to some ligands and activate key signaling pathways is different in vitro and in vivo. This raises questions about whether in vitro assays will recapitulate all aspects of the in vivo glial injury response and how in vitro findings will translate into promoting regeneration in vivo. Thus, caution is warranted in interpreting results obtained using Müller glial cell lines. Research to promote clinically relevant levels of retinal regeneration from mammalian Müller glia will benefit from the use of more complex model systems and such as ex vivo retinal explants and threedimensional substrates and the inclusion of multiple retinal cell types to better model the retinal environment.

\section{Competing Interests}

The authors declare that there is no conflict of interests regarding the publication of this paper.

\section{Acknowledgments}

This work was supported, in part, by grants from the National Institute of Health, R01-EY021792 (Deborah C. Otteson), T35-EY007088 (Krista M. Beach), and P20-EY007551 (Core) and from the University of Houston. The authors thank Micah Mesko-Smith, Amanda D. Gall, and Dr. Mary Guirguis for expert technical assistance.

\section{References}

[1] W. L. Wong, X. Su, X. Li et al., "Global prevalence of age-related macular degeneration and disease burden projection for 2020 and 2040: a systematic review and meta-analysis," The Lancet Global Health, vol. 2, no. 2, pp. e106-e116, 2014.

[2] Y. C. Tham, X. Li, T. Y. Wong, H. A. Quigley, T. Aung, and C. Y. Cheng, "Global prevalence of glaucoma and projections of glaucoma burden through 2040: a systematic review and metaanalysis," Ophthalmology, vol. 121, no. 11, pp. 2081-2090, 2040.

[3] J. Yang, B. Cai, P. Glencer, Z. Li, X. Zhang, and X. Li, "Induced pluripotent stem cells and outer retinal disease," Stem Cells International, vol. 2016, Article ID 2850873, 6 pages, 2016.

[4] N. Wu, M. Doorenbos, and D. F. Chen, "Induced pluripotent stem cells: development in the ophthalmologic field," Stem Cells International, vol. 2016, Article ID 2361763, 7 pages, 2016.

[5] Q. Wang, J. H. Stern, and S. Temple, "Regenerative medicine: solution in sight," Advances in Experimental Medicine and Biology, vol. 854, pp. 543-548, 2016.

[6] V. Di Foggia, P. Makwana, R. R. Ali, and J. C. Sowden, "Induced pluripotent stem cell therapies for degenerative disease of the outer retina: disease modeling and cell replacement," Journal of Ocular Pharmacology and Therapeutics, vol. 32, no. 5, pp. 240252, 2016.

[7] X. Chamling, V. M. Sluch, and D. J. Zack, "The potential of human stem cells for the study and treatment of glaucoma," Investigative Ophthalmology \& Visual Science, vol. 57, no. 5, pp. ORSFil-ORSFi6, 2016.

[8] D. S. Sakaguchi, S. J. Van Hoffelen, and M. J. Young, "Differentiation and morphological integration of neural progenitor cells transplanted into the developing mammalian eye," Annals of the New York Academy of Sciences, vol. 995, pp. 127-139, 2003.

[9] M. J. Seiler and R. B. Aramant, "Cell replacement and visual restoration by retinal sheet transplants," Progress in Retinal and Eye Research, vol. 31, no. 6, pp. 661-687, 2012.

[10] H. Clevers, "Modeling development and disease with organoids," Cell, vol. 165, no. 7, pp. 1586-1597, 2016.

[11] S. N. Waddington, R. Privolizzi, R. Karda, and H. C. O’Neill, “A broad overview and review of CRISPR-cas technology and stem cells," Current Stem Cell Reports, vol. 2, no. 1, pp. 9-20, 2016.

[12] J. W. Streilein, N. Ma, H. Wenkel, T. Fong Ng, and P. Zamiri, "Immunobiology and privilege of neuronal retina and pigment epithelium transplants," Vision Research, vol. 42, no. 4, pp. 487495, 2002.

[13] I. J. Crane and J. Liversidge, "Mechanisms of leukocyte migration across the blood-retina barrier," Seminars in Immunopathology, vol. 30, no. 2, pp. 165-177, 2008.

[14] M. Chen and H. Xu, "Parainflammation, chronic inflammation, and age-related macular degeneration," Journal of Leukocyte Biology, vol. 98, no. 5, pp. 713-725, 2015.

[15] B. Xian and B. Huang, "The immune response of stem cells in subretinal transplantation," Stem Cell Research and Therapy, vol. 6, no. 1, article 161, 2015.

[16] D. L. Turner, E. Y. Snyder, and C. L. Cepko, "Lineageindependent determination of cell type in the embryonic mouse retina," Neuron, vol. 4, no. 6, pp. 833-845, 1990.

[17] R. W. Young, "Cell proliferation during postnatal development of the retina in the mouse," Brain Research, vol. 353, no. 2, pp. 229-239, 1985.

[18] X. Mu, X. Fu, H. Sun et al., "Ganglion cells are required for normal progenitor-cell proliferation but not cell-fate determination 
or patterning in the developing mouse retina," Current Biology, vol. 15 , no. 6, pp. 525-530, 2005.

[19] C. Cepko, "Intrinsically different retinal progenitor cells produce specific types of progeny," Nature Reviews Neuroscience, vol. 15, no. 9, pp. 615-627, 2014.

[20] D. L. Turner and C. L. Cepko, "A common progenitor for neurons and glia persists in rat retina late in development," Nature, vol. 328, no. 6126, pp. 131-136, 1988.

[21] P. R. Johns and S. S. Easter Jr., "Growth of the adult goldfish eye. II. Increase in retinal cell number," Journal of Comparative Neurology, vol. 176, no. 3, pp. 331-341, 1977.

[22] J. R. Lenkowski and P. A. Raymond, "Müller glia: stem cells for generation and regeneration of retinal neurons in teleost fish," Progress in Retinal and Eye Research, vol. 40, pp. 94-123, 2014.

[23] P. R. Johns and R. D. Fernald, "Genesis of rods in teleost fish retina,” Nature, vol. 293, no. 5828, pp. 141-142, 1981.

[24] A. Pamela Raymond, "The unique origin of rod photoreceptors in the teleost retina," Trends in Neurosciences, vol. 8, pp. 12-17, 1985.

[25] J. W. Kwan, M. J. Lee, A. F. Mack, J. F. Chiu, and R. D. Fernald, "Nonuniform distribution of cell proliferation in the adult teleost retina," Brain Research, vol. 712, no. 1, pp. 40-44, 1996.

[26] D. Julian, K. Ennis, and J. I. Korenbrot, "Birth and fate of proliferative cells in the inner nuclear layer of the mature fish retina," Journal of Comparative Neurology, vol. 394, no. 3, pp. 271-282, 1998.

[27] D. C. Otteson, P. F. Cirenza, and P. F. Hitchcock, "Persistent neurogenesis in the teleost retina: evidence for regulation by the growth-hormone/insulin-like growth factor-I axis," Mechanisms of Development, vol. 117, no. 1-2, pp. 137-149, 2002.

[28] D. L. Stenkamp, “The rod photoreceptor lineage of teleost fish," Progress in Retinal and Eye Research, vol. 30, no. 6, pp. 395-404, 2011.

[29] D. C. Otteson, A. R. D’Costa, and P. F. Hitchcock, "Putative stem cells and the lineage of rod photoreceptors in the mature retina of the goldfish," Developmental Biology, vol. 232, no. 1, pp. 62-76, 2001.

[30] D. G. Attardi and R. W. Sperry, "Preferential selection of central pathways by regenerating optic fibers," Experimental Neurology, vol. 7, no. 1, pp. 46-64, 1963.

[31] M. Jacobson and R. M. Gaze, "Selection of appropriate tectal connections by regenerating optic nerve fibers in adult goldfish," Experimental Neurology, vol. 13, no. 4, pp. 418-430, 1965.

[32] F. Lombardo, "Course and localization of mitoses during the regeneration of the retina of an adult teleost," Addademia LinceiRendtconti Scienze Fisicali Matematiche e Natureale, vol. 8, no. 53, pp. 323-327, 1972.

[33] G. E. Landreth and B. W. Agranoff, "Explant culture of adult goldfish retina: a model for the study of CNS regeneration," Brain Research, vol. 161, no. 1, pp. 39-53, 1979.

[34] W. Maier and H. Wolburg, "Regeneration of the goldfish retina after exposure to different doses of ouabain," Cell and Tissue Research, vol. 202, no. 1, pp. 99-118, 1979.

[35] P. F. Hitchcock, K. J. Lyndsey Myhr, S. S. Easter Jr., R. MangioneSmith, and D. D. Jones, "Local regeneration in the retina of the goldfish,” Journal of Neurobiology, vol. 23, no. 2, pp. 187-203, 1992.

[36] P. F. Hitchcock, "Tracer coupling among regenerated amacrine cells in the retina of the goldfish," Visual Neuroscience, vol. 14, no. 3, pp. 463-472, 1997.
[37] D. A. Cameron, H. Vafai, and J. A. White, "Analysis of dendritic arbors of native and regenerated ganglion cells in the goldfish retina," Visual Neuroscience, vol. 16, no. 2, pp. 253-261, 1999.

[38] A. F. Mensinger and M. K. Powers, "Visual function in regenerating teleost retina following cytotoxic lesioning," Visual Neuroscience, vol. 16, no. 2, pp. 241-251, 1999.

[39] D. A. Cameron and L. H. Carney, "Cell mosaic patterns in the native and regenerated inner retina of zebrafish: implications for retinal assembly," Journal of Comparative Neurology, vol. 416, no. 3, pp. 356-367, 2000.

[40] E. M. Levine, P. F. Hitchcock, E. Glasgow, and N. Schechter, "Restricted expression of a new paired-class homeobox gene in normal and regenerating adult goldfish retina," Journal of Comparative Neurology, vol. 348, no. 4, pp. 596-606, 1994.

[41] P. F. Hitchcock, R. E. Macdonald, J. T. Van DeRyt, and S. W. Wilson, "Antibodies against Pax6 immunostain amacrine and ganglion cells and neuronal progenitors, but not rod precursors, in the normal and regenerating retina of the goldfish," Journal of Neurobiology, vol. 29, no. 3, pp. 399-413, 1996.

[42] R. L. Bernardos, L. K. Barthel, J. R. Meyers, and P. A. Raymond, "Late-stage neuronal progenitors in the retina are radial Müller glia that function as retinal stem cells," Journal of Neuroscience, vol. 27, no. 26, pp. 7028-7040, 2007.

[43] S. C. Kassen, V. Ramanan, J. E. Montgomery et al., “Time course analysis of gene expression during light-induced photoreceptor cell death and regeneration in albino zebrafish," Developmental Neurobiology, vol. 67, no. 8, pp. 1009-1031, 2007.

[44] J. L. Thomas, A. H. Ranski, G. W. Morgan, and R. Thummel, "Reactive gliosis in the adult zebrafish retina," Experimental Eye Research, vol. 143, pp. 98-109, 2016.

[45] R. Thummel, S. C. Kassen, J. E. Montgomery, J. M. Enright, and D. R. Hyde, "Inhibition of Müller glial cell division blocks regeneration of the light-damaged zebrafish retina," Developmental Neurobiology, vol. 68, no. 3, pp. 392-408, 2008.

[46] R. Thummel, S. C. Kassen, J. M. Enright, C. M. Nelson, J. E. Montgomery, and D. R. Hyde, "Characterization of Müller glia and neuronal progenitors during adult zebrafish retinal regeneration," Experimental Eye Research, vol. 87, no. 5, pp. 433444, 2008.

[47] C. M. Nelson, R. A. Gorsuch, T. J. Bailey, K. M. Ackerman, S. C. Kassen, and D. R. Hyde, "Stat3 defines three populations of müller glia and is required for initiating maximal müller glia proliferation in the regenerating zebrafish retina," Journal of Comparative Neurology, vol. 520, no. 18, pp. 4294-4311, 2012.

[48] K. Ogai, S. Hisano, K. Sugitani, Y. Koriyama, and S. Kato, "Cell fate of Müller cells during photoreceptor regeneration in an $\mathrm{N}$ methyl- $N$-nitrosourea-induced retinal degeneration model of zebrafish," Advances in Experimental Medicine and Biology, vol. 854, pp. 685-692, 2016.

[49] T. S. Vihtelic and D. R. Hyde, "Light-induced rod and cone cell death and regeneration in the adult albino zebrafish (Danio rerio) retina," Journal of Neurobiology, vol. 44, no. 3, pp. 289$307,2000$.

[50] J. Wan and D. Goldman, "Retina regeneration in zebrafish," Current Opinion in Genetics and Development, vol. 40, pp. 41-47, 2016.

[51] P. Yurco and D. A. Cameron, "Responses of Müller glia to retinal injury in adult zebrafish," Vision Research, vol. 45, no. 8, pp. 9911002, 2005.

[52] P. Hitchcock, M. Ochocinska, A. Sieh, and D. Otteson, "Persistent and injury-induced neurogenesis in the vertebrate retina," 
Progress in Retinal and Eye Research, vol. 23, no. 2, pp. 183-194, 2004.

[53] J. E. Turner, R. K. Delaney, and J. E. Johnson, "Retinal ganglion cell response to nerve growth factor in the regenerating and intact visual system of the goldfishCarassius auratus," Brain Research, vol. 197, no. 2, pp. 319-330, 1980.

[54] J. T. Schmidt and D. L. Edwards, "Activity sharpens the map during the regeneration of the retinotectal projection in goldfish," Brain Research, vol. 269, no. 1, pp. 29-39, 1983.

[55] J. E. Braisted and P. A. Raymond, "Regeneration of dopaminergic neurons in goldfish retina," Development, vol. 114, no. 4, pp. 913-919, 1992.

[56] D. A. Cameron and S. S. Easter Jr., "Cone photoreceptor regeneration in adult fish retina: phenotypic determination and mosaic pattern formation," The Journal of Neuroscience, vol. 15, no. 3, part 2, pp. 2255-2271, 1995.

[57] A. E. Lindsey and M. K. Powers, "Visual behavior of adult goldfish with regenerating retina," Visual Neuroscience, vol. 24, no. 3, pp. 247-255, 2007.

[58] A. F. Mensinger and M. K. Powers, "Visual function in regenerating teleost retina following surgical lesioning," Visual Neuroscience, vol. 24, no. 3, pp. 299-307, 2007.

[59] J. E. Braisted, T. F. Essman, and P. A. Raymond, "Selective regeneration of photoreceptors in goldfish retina," Development, vol. 120, no. 9, pp. 2409-2419, 1994.

[60] P. F. Hitchcock and J. T. Vanderyt, "Regeneration of the dopamine-cell mosaic in the retina of the goldfish," Visual Neuroscience, vol. 11, no. 2, pp. 209-217, 1994.

[61] M. Joseph Phillips and D. C. Otteson, "Differential expression of neuronal genes in müller glia in two- and three-dimensional cultures," Investigative Ophthalmology and Visual Science, vol. 52, no. 3, pp. 1439-1449, 2011.

[62] M. O. Karl, S. Hayes, B. R. Nelson, K. Tan, B. Buckingham, and T. A. Reh, "Stimulation of neural regeneration in the mouse retina," Proceedings of the National Academy of Sciences of the United States of America, vol. 105, no. 49, pp. 19508-19513, 2008.

[63] M. Takeda, A. Takamiya, J.-W. Jiao et al., " $\alpha$-Aminoadipate induces progenitor cell properties of Müller glia in adult mice," Investigative Ophthalmology and Visual Science, vol. 49, no. 3, pp. 1142-1150, 2008.

[64] D. Gallina, L. Todd, and A. J. Fischer, "A comparative analysis of Müller glia-mediated regeneration in the vertebrate retina," Experimental Eye Research, vol. 123, pp. 121-130, 2014.

[65] A. Bringmann, T. Pannicke, J. Grosche et al., "Müller cells in the healthy and diseased retina," Progress in Retinal and Eye Research, vol. 25, no. 4, pp. 397-424, 2006.

[66] A. Bringmann and P. Wiedemann, "Müller glial cells in retinal disease," Ophthalmologica, vol. 227, no. 1, pp. 1-19, 2011.

[67] C. Hippert, A. B. Graca, A. C. Barber et al., "Müller glia activation in response to inherited retinal degeneration is highly varied and disease-specific," PLOS ONE, vol. 10, no. 3, Article ID e0120415, 2015.

[68] E. Ulbricht, T. Pannicke, M. Hollborn et al., "Proliferative gliosis causes mislocation and inactivation of inwardly rectifying $\mathrm{K}^{+}$ (Kir) channels in rabbit retinal glial cells," Experimental Eye Research, vol. 86, no. 2, pp. 305-313, 2008.

[69] H. Kuhrt, A. Wurm, A. Karl et al., "Müller cell gliosis in retinal organ culture mimics gliotic alterations after ischemia in vivo," International Journal of Developmental Neuroscience, vol. 26, no. 7, pp. 745-751, 2008.
[70] A. J. Fischer, G. Omar, J. Eubanks, C. R. McGuire, B. D. Dierks, and T. A. Reh, "Different aspects of gliosis in retinal Müller glia can be induced by CNTF, insulin, and FGF2 in the absence of damage," Molecular Vision, vol. 10, pp. 973-986, 2004.

[71] S. Ooto, T. Akagi, R. Kageyama et al., "Potential for neural regeneration after neurotoxic injury in the adult mammalian retina," Proceedings of the National Academy of Sciences of the United States of America, vol. 101, no. 37, pp. 13654-13659, 2004.

[72] M. A. Tackenberg, B. A. Tucker, J. S. Swift et al., "Müller cell activation, proliferation and migration following laser injury," Molecular Vision, vol. 15, pp. 1886-1896, 2009.

[73] A. J. Fischer, C. R. McGuire, B. D. Dierks, and T. A. Reh, "Insulin and fibroblast growth factor 2 activate a neurogenic program in Müller glia of the chicken retina," The Journal of Neuroscience, vol. 22, no. 21, pp. 9387-9398, 2002.

[74] L. Todd, L. I. Volkov, C. Zelinka, N. Squires, and A. J. Fischer, "Heparin-binding EGF-like growth factor (HB-EGF) stimulates the proliferation of Müller glia-derived progenitor cells in avian and murine retinas," Molecular and Cellular Neuroscience, vol. 69, pp. 54-64, 2015.

[75] L. Todd, N. Squires, L. Suarez, and A. J. Fischer, "Jak/Stat signaling regulates the proliferation and neurogenic potential of Müller glia-derived progenitor cells in the avian retina," Scientific Reports, vol. 6, article no. 35703, 2016.

[76] A. J. Fischer and T. A. Reh, "Müller glia are a potential source of neural regeneration in the postnatal chicken retina," Nature Neuroscience, vol. 4, no. 3, pp. 247-252, 2001.

[77] Q. Jian, Z. Tao, Y. Li, and Z. Q. Yin, "Acute retinal injury and the relationship between nerve growth factor, Notchl transcription and short-lived dedifferentiation transient changes of mammalian Müller cells," Vision Research, pp. 107-117, 2015.

[78] S. G. Wohl and T. A. Reh, "miR-124-9-9* potentiates Ascl1induced reprogramming of cultured Müller glia," GLIA, vol. 64, no. 5, pp. 743-762, 2016.

[79] Y. Ueki, M. S. Wilken, K. E. Cox et al., “Transgenic expression of the proneural transcription factor Ascll in Müller glia stimulates retinal regeneration in young mice," Proceedings of the National Academy of Sciences of the United States of America, vol. 112, no. 44, pp. 13717-13722, 2015.

[80] K. Lust, R. Sinn, A. Pérez Saturnino, L. Centanin, and J. Wittbrodt, "De novo neurogenesis by targeted expression of atoh7 to Müller glia cells," Development, vol. 143, no. 11, pp. 18741883, 2016.

[81] W.-T. Song, X.-Y. Zhang, and X.-B. Xia, "Atoh7 promotes the differentiation of Müller cells-derived retinal stem cells into retinal ganglion cells in a rat model of glaucoma," Experimental Biology and Medicine, vol. 240, no. 5, pp. 682-690, 2015.

[82] J. Wan, H. Zheng, Z.-L. Chen, H.-L. Xiao, Z.-J. Shen, and G.-M. Zhou, "Preferential regeneration of photoreceptor from Müller glia after retinal degeneration in adult rat," Vision Research, vol. 48, no. 2, pp. 223-234, 2008.

[83] K. Löffler, P. Schäfer, M. Völkner, T. Holdt, and M. O. Karl, “Agedependent Müller glia neurogenic competence in the mouse retina," Glia, vol. 63, no. 10, pp. 1809-1824, 2015.

[84] Y. Ueki and T. A. Reh, "EGF stimulates müller glial proliferation via a BMP-dependent mechanism," GLIA, vol. 61, no. 5, pp. 778789, 2013.

[85] C. B. Del Debbio, Q. Mir, S. Parameswaran et al., "Notch signaling activates stem cell properties of müller glia through transcriptional regulation and Skp2-mediated degradation of p27Kip1," PLoS ONE, vol. 11, no. 3, Article ID e0152025, 2016. 
[86] R. Ringuette, M. Atkins, P. S. Lagali et al., "A Notch-Gli2 axis sustains Hedgehog responsiveness of neural progenitors and Müller glia," Developmental Biology, vol. 411, no. 1, pp. 85-100, 2016.

[87] C. Conner, K. M. Ackerman, M. Lahne, J. S. Hobgood, and D. R. Hyde, "Repressing notch signaling and expressing TNF $\alpha$ are sufficient to mimic retinal regeneration by inducing müller glial proliferation to generate committed progenitor cells," Journal of Neuroscience, vol. 34, no. 43, pp. 14403-14419, 2014.

[88] K. Ghai, C. Zelinka, and A. J. Fischer, "Notch signaling influences neuroprotective and proliferative properties of mature Müller glia," The Journal of Neuroscience, vol. 30, no. 8, pp. 3101$3112,2010$.

[89] C. M. Nelson, K. M. Ackerman, P. O’Hayer, T. J. Bailey, R. A. Gorsuch, and D. R. Hyde, "Tumor necrosis factor-alpha is produced by dying retinal neurons and is required for Müller glia proliferation during zebrafish retinal regeneration," Journal of Neuroscience, vol. 33, no. 15, pp. 6524-6539, 2013.

[90] J. R. Lenkowski, Z. Qin, C. J. Sifuentes et al., "Retinal regeneration in adult zebrafish requires regulation of TGF $\beta$ signaling," GLIA, vol. 61, no. 10, pp. 1687-1697, 2013.

[91] S. Dharmarajan, Z. Gurel, S. Wang, C. M. Sorenson, N. Sheibani, and T. L. Belecky-Adams, "Bone morphogenetic protein 7 regulates reactive gliosis in retinal astrocytes and Müller glia," Molecular Vision, vol. 20, pp. 1085-1108, 2014.

[92] A. J. Fischer, M. Schmidtb, G. Omara, and T. A. Rehb, “BMP4 and CNTF are neuroprotective and suppress damage-induced proliferation of Muller glia in the retina," Molecular and Cellular Neuroscience, vol. 27, no. 4, pp. 531-542, 2004.

[93] E. Gramage, T. D’Cruz, S. Taylor, R. Thummel, and P. F. Hitchcock, "Midkine-a protein localization in the developing and adult retina of the zebrafish and its function during photoreceptor regeneration," PLoS ONE, vol. 10, no. 3, Article ID e0121789, 2015.

[94] T. Muramatsu, "Midkine, a heparin-binding cytokine with multiple roles in development, repair and diseases," Proceedings of the Japan Academy Series B: Physical and Biological Sciences, vol. 86, no. 4, pp. 410-425, 2010.

[95] A.-A. Calinescu, T. S. Vihtelic, D. R. Hyde, and P. F. Hitchcock, "Cellular expression of midkine-a and midkine-b during retinal development and photoreceptor regeneration in zebrafish," Journal of Comparative Neurology, vol. 514, no. 1, pp. 1-10, 2009.

[96] K. D. Rhee, S. Nusinowitz, K. Chao, F. Yu, D. Bok, and X.-J. Yang, "CNTF-mediated protection of photoreceptors requires initial activation of the cytokine receptor gp130 in Müller glial cells," Proceedings of the National Academy of Sciences of the United States of America, vol. 110, no. 47, pp. E4520-E4529, 2013.

[97] W. Xue, R. I. Cojocaru, V. J. Dudley, M. Brooks, A. Swaroop, and V. P. Sarthy, "Ciliary neurotrophic factor induces genes associated with inflammation and gliosis in the retina: a gene profiling study of flow-sorted, Müller cells," PLoS ONE, vol. 6, no. 5, Article ID e20326, 2011.

[98] S. C. Kassen, R. Thummel, L. A. Campochiaro, M. J. Harding, N. A. Bennett, and D. R. Hyde, "CNTF induces photoreceptor neuroprotection and Müller glial cell proliferation through two different signaling pathways in the adult zebrafish retina," Experimental Eye Research, vol. 88, no. 6, pp. 1051-1064, 2009.

[99] Y. Wang, S. B. Smith, J. M. Ogilvie, D. J. McCool, and V. Sarthy, "Ciliary neurotrophic factor induces glial fibrillary acidic protein in retinal Müller cells through the JAK/STAT signal transduction pathway," Current Eye Research, vol. 24, no. 4, pp. 305-312, 2002.
[100] J. Wan, R. Ramachandran, and D. Goldman, "HB-EGF is necessary and sufficient for müller glia dedifferentiation and retina regeneration," Developmental Cell, vol. 22, no. 2, pp. 334$347,2012$.

[101] J. Wan, X.-F. Zhao, A. Vojtek, and D. Goldman, "Retinal injury, growth factors, and cytokines converge on $\beta$-catenin and pStat 3 signaling to stimulate retina regeneration," Cell Reports, vol. 9, no. 1, pp. 285-297, 2014.

[102] K. J. Wahlin, P. A. Campochiaro, D. J. Zack, and R. Adler, "Neurotrophic factors cause activation of intracellular signaling pathways in Muller cells and other cells of the inner retina, but not photoreceptors," Investigative Ophthalmology \& Visual Science, vol. 41, no. 3, pp. 927-936, 2000.

[103] W. Cao, R. Wen, F. Li, M. M. Lavail, and R. H. Steinberg, "Mechanical injury increases bFGF and CNTF mRNA expression in the mouse retina," Experimental Eye Research, vol. 65, no. 2, pp. 241-248, 1997.

[104] R. Wen, T. Cheng, Y. Song et al., "Continuous exposure to bright light upregulates bFGF and CNTF expression in the rat retina," Current Eye Research, vol. 17, no. 5, pp. 494-500, 1998.

[105] M. Honjo, H. Tanihara, N. Kido, M. Inatani, K. Okazaki, and Y. Honda, "Expression of ciliary neurotrophic factor activated by retinal Muller cells in eyes with NMDA- and kainic acid-induced neuronal death," Investigative Ophthalmology and Visual Science, vol. 41, no. 2, pp. 552-560, 2000.

[106] W. Cao, F. Li, R. H. Steinberg, and M. M. LaVail, "Development of normal and injury-induced gene expression of aFGF, bFGF, CNTF, BDNF, GFAP and IGF-I in the rat retina," Experimental Eye Research, vol. 72, no. 5, pp. 591-604, 2001.

[107] A. J. Fischer, M. A. Scott, E. R. Ritchey, and P. Sherwood, "Mitogen-activated protein kinase-signaling regulates the ability of Müller glia to proliferate and protect retinal neurons against excitotoxicity," GLIA, vol. 57, no. 14, pp. 1538-1552, 2009.

[108] J. J. O'Shea and R. Plenge, "JAK and STAT signaling molecules in immunoregulation and immune-mediated disease," Immunity, vol. 36, no. 4, pp. 542-550, 2012.

[109] M. Haji Abdolvahab, M. R. Mofrad, and H. Schellekens, "Interferon beta: from molecular level to therapeutic effects," International Review of Cell and Molecular Biology, vol. 326, pp. 343-372, 2016.

[110] S. Pasquin, M. Sharma, and J.-F. Gauchat, "Ciliary neurotrophic factor (CNTF): new facets of an old molecule for treating neurodegenerative and metabolic syndrome pathologies," Cytokine and Growth Factor Reviews, vol. 26, no. 5, pp. 507-515, 2015.

[111] J. A. Krall, E. M. Beyer, and G. MacBeath, "High- and lowaffinity epidermal growth factor receptor-ligand interactions activate distinct signaling pathways," PLOS ONE, vol. 6, no. 1, Article ID e15945, 2011.

[112] S. Ebong, C.-R. Yu, D. A. Carper, A. B. Chepelinsky, and C. E. Egwuagu, "Activation of STAT signaling pathways and induction of suppressors of cytokine signaling (SOCS) proteins in mammalian lens by growth factors," Investigative Ophthalmology and Visual Science, vol. 45, no. 3, pp. 872-878, 2004.

[113] C. Carter-Su, J. Schwartz, and L. S. Argetsinger, "Growth hormone signaling pathways," Growth Hormone and IGF Research, vol. 28, pp. 11-15, 2016.

[114] E. S. Park, H. Kim, J. M. Suh et al., "Involvement of JAK/STAT (Janus kinase/signal transducer and activator of transcription) in the thyrotropin signaling pathway," Molecular Endocrinology, vol. 14 , no. 5, pp. 662-670, 2000. 
[115] W. M. Peterson, Q. Wang, R. Tzekova, and S. J. Wiegand, "Ciliary neurotrophic factor and stress stimuli activate the JakSTAT pathway in retinal neurons and glia," The Journal of Neuroscience, vol. 20, no. 11, pp. 4081-4090, 2000.

[116] A. Mohr, N. Chatain, T. Domoszlai et al., "Dynamics and noncanonical aspects of JAK/STAT signalling," European Journal of Cell Biology, vol. 91, no. 6-7, pp. 524-532, 2012.

[117] M.-Y. Chang, H. Son, Y.-S. Lee, and S.-H. Lee, "Neurons and astrocytes secrete factors that cause stem cells to differentiate into neurons and astrocytes, respectively," Molecular and Cellular Neuroscience, vol. 23, no. 3, pp. 414-426, 2003.

[118] A. Plotnikov, E. Zehorai, S. Procaccia, and R. Seger, "The MAPK cascades: signaling components, nuclear roles and mechanisms of nuclear translocation," Biochimica et Biophysica Acta, vol. 1813, no. 9, pp. 1619-1633, 2011.

[119] N. Walsh, K. Valter, and J. Stone, "Cellular and subcellular patterns of expression of bFGF and CNTF in the normal and light stressed adult rat retina," Experimental Eye Research, vol. 72, no. 5, pp. 495-501, 2001.

[120] H. Chen and A. J. Weber, "Expression of glial fibrillary acidic protein and glutamine synthetase by Müller cells after optic nerve damage and intravitreal application of brain-derived neurotrophic factor," Glia, vol. 38, no. 2, pp. 115-125, 2002.

[121] G. Tuccari, C. Trombetta, M. M. Giardinelli, F. Arena, and G. Barresi, "Distribution of glial fibrillary acidic protein in normal and gliotic human retina," Basic and Applied Histochemistry, vol. 30, no. 4, pp. 425-432, 1986.

[122] M. A. Kahn, C. J. Huang, A. Caruso et al., "Ciliary neurotrophic factor activates JAK/Stat signal transduction cascade and induces transcriptional expression of glial fibrillary acidic protein in glial cells," Journal of Neurochemistry, vol. 68, no. 4, pp. 1413-1423, 1997.

[123] P. Rajan, A. J. Symes, and J. S. Fink, "STAT proteins are activated by ciliary neurotrophic factor in cells of central nervous system origin," Journal of Neuroscience Research, vol. 43, no. 4, pp. 403411, 1996.

[124] K. D. Rhee, A. Ruiz, J. L. Duncan et al., "Molecular and cellular alterations induced by sustained expression of ciliary neurotrophic factor in a mouse model of retinitis pigmentosa," Investigative Ophthalmology and Visual Science, vol. 48, no. 3, pp. 1389-1400, 2007.

[125] M. Kirsch, N. Trautmann, M. Ernst, and H.-D. Hofmann, "Involvement of gp130-associated cytokine signaling in müller cell activation following optic nerve lesion," GLIA, vol. 58, no. 7, pp. 768-779, 2010.

[126] G. Groeger, F. Doonan, T. G. Cotter, and M. Donovan, "Reactive oxygen species regulate prosurvival ERK1/2 signaling and bFGF expression in gliosis within the retina," Investigative Ophthalmology and Visual Science, vol. 53, no. 10, pp. 6645-6654, 2012.

[127] S. Kaneshiro, K. Ebina, K. Shi et al., "IL-6 negatively regulates osteoblast differentiation through the SHP2/MEK2 and SHP2/Akt2 pathways in vitro," Journal of Bone and Mineral Metabolism, vol. 32, no. 4, pp. 378-392, 2014.

[128] J. M. Askvig and J. A. Watt, "The MAPK and PI3K pathways mediate CNTF-induced neuronal survival and process outgrowth in hypothalamic organotypic cultures," Journal of Cell Communication and Signaling, vol. 9, no. 3, pp. 217-231, 2015.

[129] S. Higashiyama, J. A. Abraham, J. Miller, J. C. Fiddes, and M. Klagsbrun, "A heparin-binding growth factor secreted by macrophage-like cells that is related to EGF," Science, vol. 251, no. 4996, pp. 936-939, 1991.
[130] A. J. Fischer, M. A. Scott, and W. Tuten, "Mitogen-activated protein kinase-signaling stimulates Müller glia to proliferate in acutely damaged chicken retina," GLIA, vol. 57, no. 2, pp. 166181, 2009.

[131] Y. Ueki, M. O. Karl, S. Sudar et al., "P53 is required for the developmental restriction in Müller glial proliferation in mouse retina," Glia, vol. 60, no. 10, pp. 1579-1589, 2012.

[132] A. Suga, K. Sadamoto, M. Fujii, M. Mandai, and M. Takahashi, "Proliferation potential of Müller glia after retinal damage varies between mouse strains," PLoS ONE, vol. 9, no. 4, Article ID e94556, 2014.

[133] D. C. Otteson and M. J. Phillips, "A conditional immortalized mouse müller glial cell line expressing glial and retinal stem cell genes," Investigative Ophthalmology and Visual Science, vol. 51, no. 11, pp. 5991-6000, 2010.

[134] J. M. Lawrence, S. Singhal, B. Bhatia et al., "MIO-M1 cells and similar Müller glial cell lines derived from adult human retina exhibit neural stem cell characteristics," Stem Cells, vol. 25, no. 8, pp. 2033-2043, 2007.

[135] V. P. Sarthy, S. J. Brodjian, K. Dutt, B. N. Kennedy, R. P. French, and J. W. Crabb, "Establishment and characterization of a retinal Müller cell line," Investigative Ophthalmology \& Visual Science, vol. 39, no. 1, pp. 212-216, 1998.

[136] M. Tomi, T. Funaki, H. Abukawa et al., "Expression and regulation of L-cystine transporter, system $\mathrm{X}_{c}^{-}$, in the newly developed rat retinal Müller cell line (TR-MUL)," GLIA, vol. 43, no. 3, pp. 208-217, 2003.

[137] M. Hollborn, E. Ulbricht, K. Rillich et al., “The human Müller cell line MIO-M1 expresses opsins," Molecular Vision, vol. 17, pp. 2738-2750, 2011.

[138] S. M. Hauck, S. Suppmann, and M. Ueffing, "Proteomic profiling of primary retinal Muller glia cells reveals a shift in expression patterns upon adaptation to in vitro conditions," Glia, vol. 44, no. 3, pp. 251-263, 2003.

[139] S. Singhal, B. Bhatia, H. Jayaram et al., "Human müller glia with stem cell characteristics differentiate into retinal ganglion cell (RGC) precursors in vitro and partially restore RGC function in vivo following transplantation," Stem Cells Translational Medicine, vol. 1, no. 3, pp. 188-199, 2012.

[140] H. Jayaram, M. F. Jones, K. Eastlake et al., "Transplantation of photoreceptors derived from human Müller glia restore rod function in the $\mathrm{P} 23 \mathrm{H}$ rat," Stem Cells Translational Medicine, vol. 3, no. 3, pp. 323-333, 2014.

[141] W.-T. Song, X.-Y. Zhang, and X.-B. Xia, "Atoh7 promotes the differentiation of retinal stem cells derived from Müller cells into retinal ganglion cells by inhibiting Notch signaling," Stem Cell Research and Therapy, vol. 4, no. 4, article 94, 2013.

[142] J. Pollak, M. S. Wilken, Y. Ueki et al., "ASCL1 reprograms mouse Müller glia into neurogenic retinal progenitors," Development, vol. 140, no. 12, pp. 2619-2631, 2013.

[143] K. Beach and O. Dc, "Culture conditions, but not CNTF, modulate ERK/STAT signaling and gene expression in mouse Müller glia in vitro," Investigative Ophthalmology \& Visual Science, vol. 56, abstract 5492, 2016.

[144] S. Bhattacharya, A. V. Das, K. B. Mallya, and I. Ahmad, "Ciliary neurotrophic factor-mediated signaling regulates neuronal versus glial differentiation of retinal stem cells/progenitors by concentration-dependent recruitment of mitogen-activated protein kinase and janus kinase-signal transducer and activator of transcription pathways in conjunction with notch signaling," Stem Cells, vol. 26, no. 10, pp. 2611-2624, 2008. 
[145] O. Goureau, K. D. Rhee, and X.-J. Yang, "Ciliary neurotrophic factor promotes Müller glia differentiation from the postnatal retinal progenitor pool," Developmental Neuroscience, vol. 26, no. 5-6, pp. 359-370, 2004.

[146] S. S.-M. Zhang, J. Wei, H. Qin et al., "STAT3-mediated signaling in the determination of rod photoreceptor cell fate in mouse retina," Investigative Ophthalmology and Visual Science, vol. 45, no. 7, pp. 2407-2412, 2004.

[147] K. D. Rhee, O. Goureau, S. Chen, and X.-J. Yang, "Cytokineinduced activation of signal transducer and activator of transcription in photoreceptor precursors regulates rod differentiation in the developing mouse retina," The Journal of Neuroscience, vol. 24, no. 44, pp. 9779-9788, 2004.

[148] A. F. Bray, R. R. Cevallos, K. Gazarian, and M. Lamas, "Human dental pulp stem cells respond to cues from the rat retina and differentiate to express the retinal neuronal marker rhodopsin," Neuroscience, vol. 280, pp. 142-155, 2014.

[149] M. Foldvari and D. W. Chen, "The intricacies of neurotrophic factor therapy for retinal ganglion cell rescue in glaucoma: a case for gene therapy," Neural Regeneration Research, vol. 11, no. 6, pp. 875-877, 2016.

[150] X. Xia and I. Ahmad, "Unlocking the neurogenic potential of mammalian müller glia," International Journal of Stem Cells, vol. 9, no. 2, pp. 169-175, 2016.

[151] A. Alunni and L. Bally-Cuif, "A comparative view of regenerative neurogenesis in vertebrates," Development, vol. 143, no. 5, pp. 741-753, 2016.

[152] S. Ghosh and S. P. Hui, "Regeneration of zebrafish CNS: adult neurogenesis," Neural Plasticity, vol. 2016, Article ID 5815439, 2016.

[153] H. K. Yip, "Retinal stem cells and regeneration of vision system," Anatomical Record, vol. 297, no. 1, pp. 137-160, 2014.

[154] D. Goldman, "Müller glial cell reprogramming and retina regeneration," Nature Reviews Neuroscience, vol. 15, no. 7, pp. 431-442, 2014.

[155] H. Liu, G. Liu, and Y. Bi, "CNTF regulates neurite outgrowth and neuronal migration through JAK2/STAT3 and PI3K/Akt signaling pathways of DRG explants with gp120-induced neurotoxicity in vitro," Neuroscience Letters, vol. 569, pp. 110-115, 2014.

[156] M. Inoue, C. Nakayama, and H. Noguchi, "Activating mechanism of CNTF and related cytokines," Molecular Neurobiology, vol. 12, no. 3, pp. 195-209, 1996.

[157] K. Ikeda, T. Tatsuno, H. Noguchi, and C. Nakayama, "Ciliary neurotrophic factor protects rat retina cells in vitro and in vivo via PI3 kinase," Current Eye Research, vol. 29, no. 4-5, pp. 349$355,2004$.

[158] T. Alonzi, G. Middleton, S. Wyatt et al., "Role of STAT3 and PI 3-kinase/Akt in mediating the survival actions of cytokines on sensory neurons," Molecular and Cellular Neuroscience, vol. 18, no. 3, pp. 270-282, 2001.

[159] T. G. Boulton, N. Stahl, and G. D. Yancopoulos, "Ciliary neurotrophic factor/leukemia inhibitory factor/interleukin 6/oncostatin $\mathrm{M}$ family of cytokines induces tyrosine phosphorylation of a common set of proteins overlapping those induced by other cytokines and growth factors," The Journal of Biological Chemistry, vol. 269, no. 15, pp. 11648-11655, 1994.

[160] S. M. Taylor, K. Alvarez-Delfin, C. J. Saade et al., “The bHLH transcription factor neuroD governs photoreceptor genesis and regeneration through delta-notch signaling," Investigative Ophthalmology and Visual Science, vol. 56, no. 12, pp. 7496-7515, 2015.
[161] B. Liu, D. J. Hunter, S. Rooker et al., "Wnt signaling promotes Müller cell proliferation and survival after injury," Investigative Opthalmology \& Visual Science, vol. 54, no. 1, pp. 444-453, 2013.

[162] R. Ramachandran, X.-F. Zhao, and D. Goldman, "Asclla/Dkk/ $\beta$ catenin signaling pathway is necessary and glycogen synthase kinase- $3 \beta$ inhibition is sufficient for zebrafish retina regeneration," Proceedings of the National Academy of Sciences of the United States of America, vol. 108, no. 38, pp. 15858-15863, 2011.

[163] A. Angbohang, N. Wu, T. Charalambous et al., "Downregulation of the canonical WNT signaling pathway by TGF $\beta 1$ inhibits photoreceptor differentiation of adult human Müller Glia with stem cell characteristics," Stem Cells and Development, vol. 25, no. 1, pp. 1-12, 2016.

[164] T. Haynes, C. Gutierrez, J.-C. Aycinena, P. A. Tsonis, and K. Del Rio-Tsonis, "BMP signaling mediates stem/progenitor cell-induced retina regeneration," Proceedings of the National Academy of Sciences of the United States of America, vol. 104, no. 51, pp. 20380-20385, 2007.

[165] G. Das, A. M. Clark, and E. M. Levine, "Cyclin D1 inactivation extends proliferation and alters histogenesis in the postnatal mouse retina," Developmental Dynamics, vol. 241, no. 5, pp. 941952, 2012.

[166] X. Xia and I. Ahmad, "Let-7 microRNA regulates neurogliogenesis in the mammalian retina through Hmga2," Developmental Biology, vol. 410, no. 1, pp. 70-85, 2016.

[167] K. Rajaram, R. L. Harding, T. Bailey, J. G. Patton, and D. R. Hyde, "Dynamic miRNA expression patterns during retinal regeneration in zebrafish: reduced dicer or miRNA expression suppresses proliferation of Müller Glia-derived neuronal progenitor cells," Developmental Dynamics, vol. 243, no. 12, pp. 1591-1605, 2014.

[168] K. Rajaram, R. L. Harding, D. R. Hyde, and J. G. Patton, “ $m i R-$ 203 regulates progenitor cell proliferation during adult zebrafish retina regeneration," Developmental Biology, vol. 392, no. 2, pp. 393-403, 2014.

[169] E. Gramage, J. Li, and P. Hitchcock, "The expression and function of midkine in the vertebrate retina," British Journal of Pharmacology, vol. 171, no. 4, pp. 913-923, 2014.

[170] Y. Yoshida, H. Sakakima, F. Matsuda, and M. Ikutomo, "Midkine in repair of the injured nervous system," British Journal of Pharmacology, vol. 171, no. 4, pp. 924-930, 2014.

[171] C. Xu, S. Zhu, M. Wu, W. Han, and Y. Yu, "Functional receptors and intracellular signal pathways of midkine $(\mathrm{MK})$ and pleiotrophin (PTN)," Biological and Pharmaceutical Bulletin, vol. 37, no. 4, pp. 511-520, 2014.

[172] C. Winkler and S. Yao, "The midkine family of growth factors: diverse roles in nervous system formation and maintenance," British Journal of Pharmacology, vol. 171, no. 4, pp. 905-912, 2014.

[173] L. Haynes and M. Rumsby, "The pleiotropin/midkine family of cytokines: role in glial-neuronal signalling," Progress in Brain Research, vol. 132, pp. 313-324, 2001.

[174] N. Zhang and T. F. Deuel, "Pleiotrophin and midkine, a family of mitogenic and angiogenic heparin-binding growth and differentiation factors," Current Opinion in Hematology, vol. 6, no. 1, pp. 44-50, 1999.

[175] K. Unoki, N. Ohba, H. Arimura, H. Muramatsu, and T. Muramatsu, "Rescue of photoreceptors from the damaging effects of constant light by midkine, a retinoic acid-responsive gene product," Investigative Ophthalmology \& Visual Science, vol. 35, no. 12, pp. 4063-4068, 1994. 
[176] K. Masuda, I. Watanabe, K. Unoki, N. Ohba, and T. Muramatsu, "Functional rescue of photoreceptors from the damaging effects of constant light by survival-promoting factors in the rat," Investigative Ophthalmology and Visual Science, vol. 36, no. 10, pp. 2142-2146, 1995.

[177] M. Zarbin, "Cell-based therapy for degenerative retinal disease," Trends in Molecular Medicine, vol. 22, no. 2, pp. 115-134, 2016.

[178] D. A. Parfitt, A. Lane, C. Ramsden et al., "Using induced pluripotent stem cells to understand retinal ciliopathy disease mechanisms and develop therapies," Biochemical Society Transactions, vol. 44, no. 5, pp. 1245-1251, 2016.

[179] Y. Li, L. Chan, H. V. Nguyen, and S. H. Tsang, "Personalized medicine: cell and gene therapy based on patient-specific iPSC-derived retinal pigment epithelium cells," Advances in Experimental Medicine and Biology, vol. 854, pp. 549-555, 2016.

[180] H. Klassen, "Stem cells in clinical trials for treatment of retinal degeneration," Expert Opinion on Biological Therapy, vol. 16, no. 1, pp. 7-14, 2016.

[181] S. Khan, S. S.-C. Hung, and R. C.-B. Wong, "The use of induced pluripotent stem cells for studying and treating optic neuropathies," Current Opinion in Organ Transplantation, vol. 21, no. 5, pp. 484-489, 2016.

[182] A. Zheng, Y. Li, and S. H. Tsang, "Personalized therapeutic strategies for patients with retinitis pigmentosa," Expert Opinion on Biological Therapy, vol. 15, no. 3, pp. 391-402, 2015.

[183] L. A. Wiley, E. R. Burnight, A. E. Songstad et al., "Patientspecific induced pluripotent stem cells (iPSCs) for the study and treatment of retinal degenerative diseases," Progress in Retinal and Eye Research, vol. 44, pp. 15-35, 2015.

[184] L. S. Wright, M. J. Phillips, I. Pinilla, D. Hei, and D. M. Gamm, "Induced pluripotent stem cells as custom therapeutics for retinal repair: progress and rationale," Experimental Eye Research, vol. 123, pp. 161-172, 2014. 

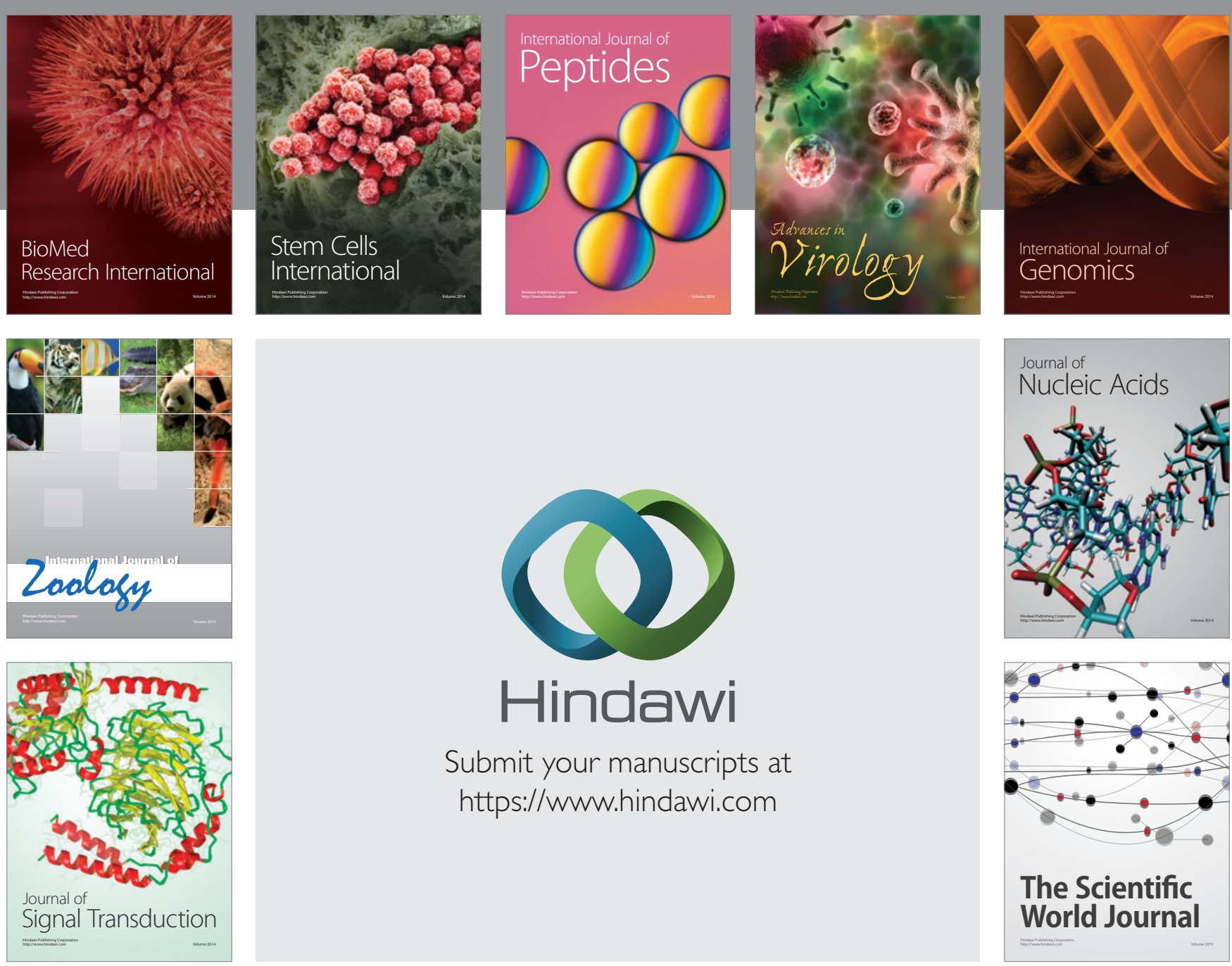

Submit your manuscripts at

https://www.hindawi.com
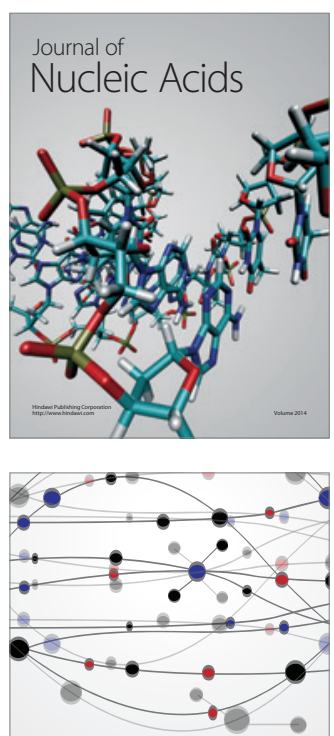

The Scientific World Journal
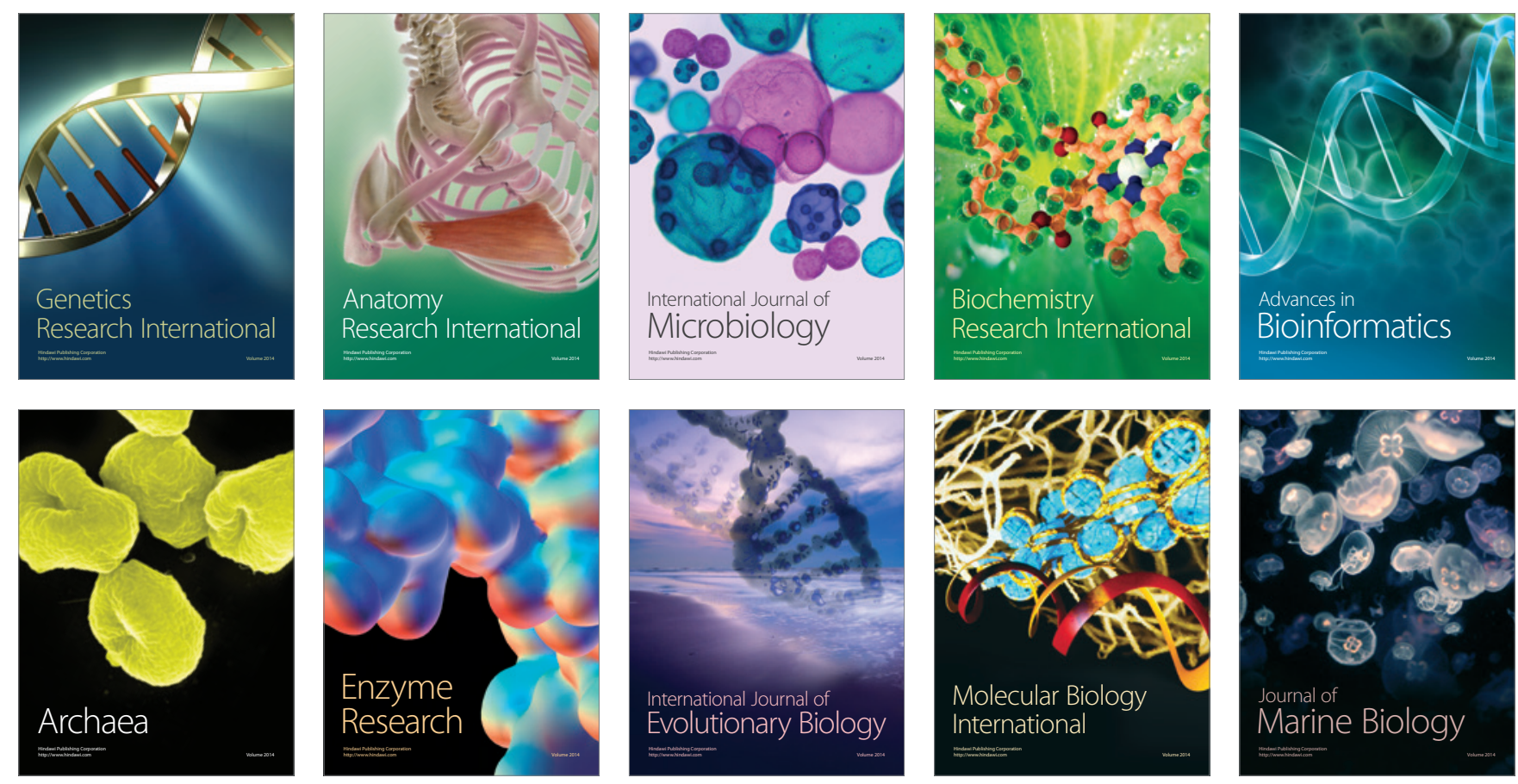